DOI 10.4171/JEMS/238

Sergey Fomin · Grigory Mikhalkin

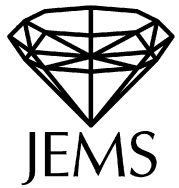

\title{
Labeled floor diagrams for plane curves
}

Received August 19, 2009 and in revised form January 19, 2010

\begin{abstract}
Floor diagrams are a class of weighted oriented graphs introduced by E. Brugallé and the second author. Tropical geometry arguments lead to combinatorial descriptions of (ordinary and relative) Gromov-Witten invariants of projective spaces in terms of floor diagrams and their generalizations. In a number of cases, these descriptions can be used to obtain explicit (direct or recursive) formulas for the corresponding enumerative invariants. In particular, we use this approach to enumerate rational curves of given degree passing through a collection of points on the complex plane and having maximal tangency to a given line. Another application of the combinatorial approach is a proof of a conjecture by P. Di Francesco-C. Itzykson and L. Göttsche that in the case of a fixed cogenus, the number of plane curves of degree $d$ passing through suitably many generic points is given by a polynomial in $d$, assuming that $d$ is sufficiently large. Furthermore, the proof provides a method for computing these "node polynomials".

A labeled floor diagram is obtained by labeling the vertices of a floor diagram by the integers $1, \ldots, d$ in a manner compatible with the orientation. We show that labeled floor diagrams of genus 0 are equinumerous to labeled trees, and therefore counted by the celebrated Cayley formula. The corresponding bijections lead to interpretations of the Kontsevich numbers (the genus-0 GromovWitten invariants of the projective plane) in terms of certain statistics on trees.
\end{abstract}

Keywords. Gromov-Witten invariant, tropical curve, floor diagram, labeled tree

\section{Contents}

1. Labeled floor diagrams and their markings . . . . . . . . . . . . . . . . . . . . . . 1456

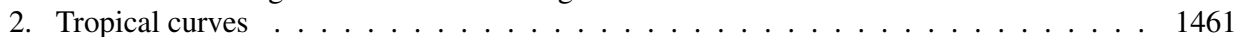

3. The combinatorial correspondence theorems . . . . . . . . . . . . . . . . . . . . . . . . . . . 1464

4. Computing relative Gromov-Witten invariants . . . . . . . . . . . . . . . . . . . 1472

5. Node polynomials . . . . . . . . . . . . . . . . . . . . . . . . . . . . . . 1479

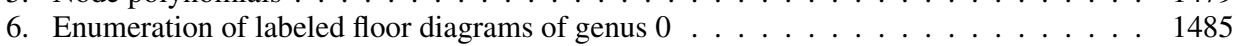

7. Conjectures and open problems . . . . . . . . . . . . . . . . . . . . . . . . . 1488

8. Welschinger invariants and odd floor diagrams . . . . . . . . . . . . . . . . . . 1491

Appendix A: Labeled floor diagrams with $d \leq 4 . \ldots$. . . . . . . . . . . . . . . . . . . . . 1492

Appendix B: Tropical rational cubics and their marked floor diagrams . . . . . . . . . . . 1494

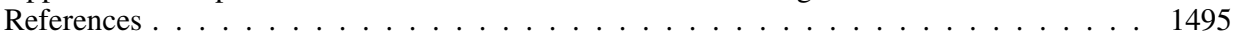

S. Fomin: Department of Mathematics, University of Michigan, Ann Arbor, MI 48109, USA; e-mail: fomin@umich.edu

G. Mikhalkin: Section de mathématiques, Université de Genève, 1211 Geneva, Switzerland; e-mail: grigory.mikhalkin@unige.ch

Mathematics Subject Classification (2010): Primary 14N10; Secondary 05A15, 14N35 


\section{Introduction}

The primary purpose of this paper is to advertise a general paradigm for solving a large class of problems of classical enumerative geometry. Although the main ingredients of the approach described herein have already appeared in the literature, a coherent presentation, complete with new convincing applications, has been lacking. Our goal is to fill the gap, and in doing so win over a few converts.

A typical problem of enumerative geometry asks for the number of geometric objects, say complex algebraic varieties of specified kind, which satisfy a number of incidence or tangency constraints. In many cases (admittedly subject to limitations, both technical and intrinsic), one can reduce such a problem to its tropical counterpart, that is, to the problem of weighted enumeration of certain polyhedral complexes known as tropical varieties. This reduction constitutes the first phase of a solution.

The goal of the second phase, which can be called discretization, is to replace piecewise-linear objects of tropical geometry by purely combinatorial ones. If this is done successfully, one obtains a manifestly positive combinatorial rule (similar in spirit to the various Littlewood-Richardson-type rules in Schubert Calculus) that identifies the answer to the original geometric problem as the number of combinatorial objects of a particular, complicated but explicit, kind.

The third and final phase is one of purely combinatorial enumeration of the relevant discrete objects. Ideally, albeit seldom, it yields a formula for the numbers in question, or some associated generating function. Otherwise, a recursion would do, or else an equation (algebraic, differential, or functional) for the generating function. At the very least, one would like to relate the objects to be enumerated to some more familiar combinatorial gadgets, placing the problem within a well developed context.

In this paper, we discuss this approach as it applies to the problem of enumerating plane complex algebraic curves with given properties, or more precisely, the problem of computing the Gromov-Witten invariants, both ordinary and relative, of the complex projective plane $\mathbb{P}^{2}$. Recall that the Gromov-Witten invariant $N_{d, g}$ is the number of irreducible curves of degree $d$ and genus $g$ passing through a fixed generic configuration of $3 d+g-1$ points on $\mathbb{P}^{2}$. A more general relative Gromov-Witten invariant $N_{d, g}(\lambda, \rho)$ is the number of such curves which, besides passing through appropriately many generic points, satisfy tangency conditions with respect to a given line $L$. The two integer partitions $\lambda$ and $\rho$ describe the degrees of tangency at two generic collections of points on $L$; the points in the first collection are fixed while those in the second one are allowed to vary along $L$.

The tropical reduction for the problem of computing the Gromov-Witten invariants $N_{d, g}$ (resp., $N_{d, g}(\lambda, \rho)$ ) is accomplished by means of the correspondence theorem established in [27] (resp., [29]); see Theorem 3.8 (resp., Theorem 3.12). Even though this is the most substantial step among the three required for a solution, we discuss it in less detail as the topic is already well covered in the existing literature.

For the problem of computing the invariants $N_{d, g}$, the discretization reduction has recently been given by E. Brugallé and the second author [8] (see also an excellent exposition [7], in French), by establishing a bijection between the tropical curves in ques- 
tion and certain markings ("decorations") of a particular kind of weighted acyclic graphs called floor diagrams; see Theorem 3.7. This result extends to the relative setting as well; see Theorem 3.17

Apart from a review of the aforementioned results, the bulk of this paper is dedicated to the "third phase" of combinatorial enumeration, as it applies to the problem at hand. Even though the general problem of (weighted) enumeration of marked floor diagrams appears too unwieldy to allow for an explicit solution, the latter can be achieved in a number of particular instances. In Theorem 4.10, we enumerate irreducible rational curves of given degree passing through a collection of points on the complex plane and having maximal tangency to a given line; the point of tangency can be either prescribed or left unspecified. We also compute the relative Gromov-Witten invariants associated with nonsingular or uninodal curves (Corollaries 4.4 and 4.5), and with curves passing through a triple of collinear points (Corollary 4.7$)$.

If the number of nodes $\delta=(d-1)(d-2) / 2-g$ is fixed while $d$ varies, determining $N_{d, g}$ as a function of $d$ is a classical problem with venerable history; see Section 5 and references therein. In 1994, P. Di Francesco and C. Itzykson hypothesized [14] that, for $\delta$ fixed and $d$ sufficiently large, the Gromov-Witten invariant $N_{d, g}$ (or equivalently the corresponding Severi degree) is a polynomial in $d$ (necessarily of degree $2 \delta$ ). A more explicit version of this conjecture was proposed by L. Göttsche [19]. The cases $\delta \leq 6$ of Göttsche's conjecture were established by I. Vainsencher [41]; then S. Kleiman and R. Piene [22] extended these results to $\delta \leq 8$. In Section 5, we give a proof of this conjecture based on the combinatorial rule of Theorem 1.6. (Alternative proofs have been proposed in unpublished preprints by Y. Choi [13], A.-K. Liu [26], and Y.-J. Tzeng [40].) We stress that our proof does not merely establish polynomiality of Severi degrees: it provides a method for computing those "node polynomials" explicitly and directly, without resorting to interpolation. Our method has been implemented, with a few improvements, by F. Block [6], who computed the node polynomials for all $\delta \leq 13$.

We reformulate the rule given in [8] in the language of labeled floor diagrams obtained by labeling the vertices of a floor diagram of degree $d$ by the integers $1, \ldots, d$ in a manner compatible with orientation. This point of view, applied consistently throughout the paper, is not merely a matter of language or convenience. In Theorem 6.1. we show that labeled floor diagrams of degree $d$ and genus $g=0$ are equinumerous to labeled trees on $d$ vertices, and hence counted by the celebrated Cayley formula. The corresponding bijections between labeled floor diagrams and trees yield interpretations of the numbers $N_{d, 0}$ in terms of certain statistics on trees.

Two well known recursive formulas for the Gromov-Witten invariants of the projective plane are due to M. Kontsevich [23] (for $N_{d, 0}$ ) and to L. Caporaso and J. Harris [10] (for $N_{d, g}(\lambda, \rho)$ ), respectively; see Section 7.3 Each of these formulas can in principle be obtained directly from the corresponding combinatorial rule (Theorem 1.6 and Theorem 3.18, respectively). While the derivation of the Caporaso-Harris recursion is relatively straightforward (see [2, 7]), deducing Kontsevich's formula (7.2) seems to require substantial technical effort aimed at replicating the original argument of Kontsevich in a purely combinatorial setting. 
The paper by Caporaso and Harris contains two recursive formulas: the recursion [10, Theorem 1.1] for the number of all (not necessarily irreducible) curves with given properties, and another recursion in [10, Section 1.4] for the relative Gromov-Witten numbers $N_{d, g}(\lambda, \rho)$. The first recursion does not lead to a manifestly positive rule for the numbers $N_{d, g}(\lambda, \rho)$ as it has to be followed by an involved inclusion-exclusion procedure to restrict the count to irreducible curves. The second recursion can in principle be used to obtain a positive rule, albeit an exceedingly cumbersome one.

The paper is organized as follows. Section 1 introduces labeled floor diagrams, and culminates in a reformulation of the main result of [8] (see Theorem 1.6) in this language. Section 2 reviews the basics of plane tropical curves. Section 3 discusses various versions of the correspondence theorems, both geometric and combinatorial. Applications to computation of relative Gromov-Witten invariants are presented in Section 4. The proof of the polynomiality conjecture of Di Francesco-Itzykson and Göttsche is given in Section 5 Section 6 is devoted to enumeration of labeled floor diagrams and related objects. In Section 7, we formulate a number of conjectures and open problems. In Section 8 , we briefly discuss the related problem of determining the Welschinger invariants for real plane algebraic curves.

In order to make the text accessible to both algebraic geometers and enumerative combinatorialists, we tried to make it as self-contained as possible, and in particular introduce the basic relevant background without referring to outside sources.

\section{Labeled floor diagrams and their markings}

\subsection{Preliminaries on floor diagrams}

We use standard combinatorial terminology; see, e.g., [24, 37, 38].

Definition 1.1 (Labeled floor diagram and its multiplicity). Let $d>0$ and $g \geq 0$ be integers. A (connected) labeled floor diagram $\mathcal{D}$ of degree $d$ and genus $g$ is a connected oriented graph $G=(V, E)$ on a linearly ordered $d$-element vertex set $V$ together with a weight function $w: E \rightarrow \mathbb{Z}_{>0}$ such that the following conditions are satisfied:

- (Genus) The edge set $E$ consists of $d+g-1$ edges. Equivalently, the first Betti number of $G$ is equal to $g$, assuming that $G$ is stripped of orientation and viewed as a topological space (a 1-dimensional simplicial complex).

- (Compatibility with linear ordering on $V$ ) Each edge in $E$ is directed from a vertex $u$ to a vertex $v>u$. Thus $G$ is acyclic, and in particular has no loops. Multiple edges are allowed.

- (Divergence) For each vertex $v \in V$, we have

$$
\operatorname{div}(v):=\sum_{v \stackrel{e}{\longrightarrow} \circ} w(e)-\sum_{\circ \stackrel{e}{\longrightarrow} v} w(e) \leq 1,
$$

where the first sum (respectively, the second one) is over all edges $e$ directed away from $v$ (respectively, towards $v$ ). 
The number

$$
\mu(\mathcal{D})=\mu^{\mathbb{C}}(\mathcal{D})=\prod_{e \in E}(w(e))^{2}
$$

is called the (complex) multiplicity of a labeled floor diagram $\mathcal{D}$.

Remark 1.2. An (unlabeled) floor diagram, introduced in [8] (in a more general setting of curves in $\mathbb{C P}^{n}$ ), is essentially a labeled floor diagram considered up to an isomorphism of weighted oriented graphs. There are also other discrepancies with the setup in [8], but they can be viewed as a matter of convention. In this paper, we work exclusively with labeled floor diagrams. In our opinion, this approach is more natural from a combinatorial perspective.

All labeled floor diagrams with $\leq 4$ vertices are listed in Appendix A. Each vertex set is ordered left to right; each edge is oriented towards the right.

Example 1.3. An example of a labeled floor diagram $\mathcal{D}$ is shown below:

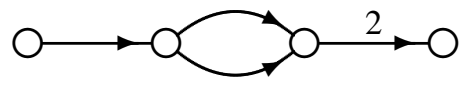

This labeled floor diagram has degree $d=4$ and genus $g=1$. It has $d=4$ vertices, $d+g-1=4$ edges, vertex divergencies $\operatorname{div}(v)$ equal to $1,1,0,-2$, respectively, and multiplicity $\mu(\mathcal{D})=4$.

Definition 1.4 (Marking of a labeled floor diagram). Let $\mathcal{D}$ be a labeled floor diagram of degree $d$ and genus $g$, as in Definition 1.1 A marking of $\mathcal{D}$ is a "combinatorial decoration" of $\mathcal{D}$ obtained by the following procedure. (We will illustrate the steps of this procedure using the diagram from Example 1.3 .)

Step 1. For each vertex $v \in V$, introduce $1-\operatorname{div}(v)$ (cf. (1.1) new distinct vertices, and connect $v$ with each of them by a single edge directed away from $v$ :

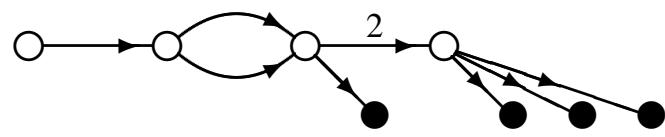

Step 2. Split each original edge $e \in E$ in two, by inserting an extra vertex in the middle of $e$; the resulting two edges inherit $e$ 's orientation and weight:

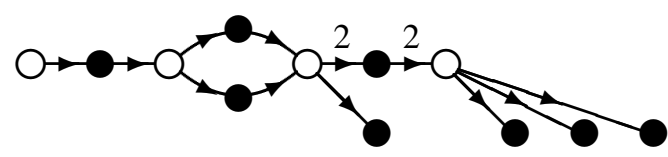

Let $\widetilde{G}=(\widetilde{V}, \widetilde{E})$ denote the acyclic directed graph obtained after Steps $1-2$. It is easy to see that $\widetilde{G}$ has $3 d+g-1$ vertices and $3 d+2 g-2$ edges.

Step 3. Extend the linear ordering on $V$ to $\widetilde{V}$ so that, as before, each edge in $\widetilde{E}$ is directed from a smaller to a larger vertex: 


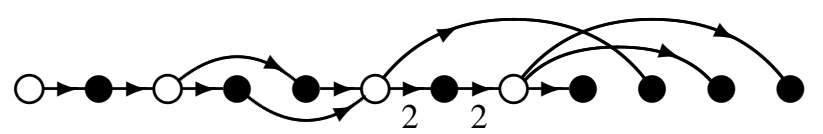

The resulting object $\widetilde{\mathcal{D}}$ is called a marked floor diagram, or a marking of the original labeled floor diagram $\mathcal{D}$. Thus $\widetilde{\mathcal{D}}$ is a directed graph $\widetilde{G}=(\widetilde{V}, \widetilde{E})$ as above, together with a linear order on $\widetilde{V}$ that extends the linear order on $V$. More precisely, we consider $\widetilde{\mathcal{D}}$ up to an automorphism that fixes $V$, so that linear orderings on $\widetilde{V}$ which produce isomorphic weighted directed graphs are viewed as the same marking of $\mathcal{D}$.

The number of markings of $\mathcal{D}$ is denoted by $v(\mathcal{D})$.

Example 1.5. In our running example (see $(1.3)$ ), we have $v(\mathcal{D})=6$. These six markings can be obtained from the diagram in (1.4) by relocating the right endpoint of the edge connecting the 6th vertex (counting from the left) to the 10th vertex to any of the five alternative positions.

Note that switching the 4 th and 5 th vertices does not change the marking since it produces an isomorphic object.

For many more examples, see Appendix A.

Since the vertex set $\widetilde{V}$ is linearly ordered, it is convenient to identify it with $\{1, \ldots$, $3 d+g-1\}$. (This also takes care of the isomorphism issue.) Also, we do not have to indicate the orientation of the edges once the ordering of vertices has been fixed. So for example the marked floor diagram $\widetilde{\mathcal{D}}$ above can just as well be drawn without the arrows:

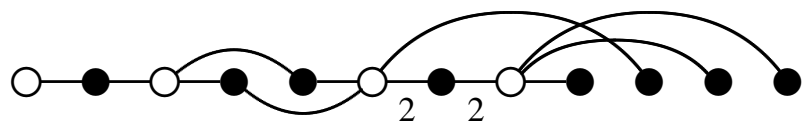

We note that even though the underlying graph $\widetilde{G}$ of a marked floor diagram $\widetilde{\mathcal{D}}$ is naturally a Hasse diagram of a partially ordered set, it would be incorrect to define the markings of $\mathcal{D}$ simply as linear extensions of this poset because such a definition would ignore the condition of compatibility of a linear extension with the original linear order on $V$.

\subsection{Combinatorial rules for Gromov-Witten invariants and Severi degrees}

The following result can be seen to be a restatement, in the language introduced above, of the first claim in [8, Theorem 1].

Theorem 1.6. The Gromov-Witten invariant $N_{d, g}$ is equal to

$$
N_{d, g}=\sum_{\mathcal{D}} \mu(\mathcal{D}) v(\mathcal{D})
$$

the sum over all labeled floor diagrams $\mathcal{D}$ of degree $d$ and genus $g$.

To rephrase, the number $N_{d, g}$ is obtained by enumerating marked floor diagrams $\widetilde{\mathcal{D}}$ of degree $d$ and genus $g$, each taken with its multiplicity $\mu(\widetilde{\mathcal{D}})=\mu(\mathcal{D})$. 
The origins of Theorem 1.6 lie in the realm of tropical geometry. They are discussed in Section 3.1. following a review of the relevant background on tropical curves in Section 2

Example 1.7. The values of $\mu(\mathcal{D})$ and $v(\mathcal{D})$ for all diagrams $\mathcal{D}$ with $d \leq 4$ are listed in Appendix A. The formula (1.5) then gives:

$N_{1,0}=1 \cdot 1=1$ (unique line through two generic points in $\mathbb{C}^{2}$ ),

$N_{2,0}=1 \cdot 1=1$ (unique conic through five generic points in $\mathbb{C}^{2}$ ),

$N_{3,0}=1 \cdot 5+4 \cdot 1+1 \cdot 3=12$ (rational cubics through eight generic points in $\mathbb{C}^{2}$ ),

$N_{4,0}=1 \cdot 40+4 \cdot 8+\cdots+1 \cdot 15=620$ (rational quartics, 11 generic points in $\mathbb{C}^{2}$ ),

$N_{3,1}=1 \cdot 1=1$ (unique cubic through nine generic points in $\mathbb{C}^{2}$ ),

$N_{4,1}=1 \cdot 26+4 \cdot 4+\cdots+1 \cdot 6=225$ (elliptic quartics, 12 generic points in $\mathbb{C}^{2}$ ),

$N_{4,2}=1 \cdot 3+1 \cdot 5+\cdots+4 \cdot 2=27$ (genus 2 quartics, 13 generic points in $\mathbb{C}^{2}$ ),

$N_{4,3}=1 \cdot 1=1$ (unique quartic through 14 generic points in $\mathbb{C}^{2}$ ).

Several approaches have been suggested to the computation of the Gromov-Witten numbers $N_{d, g}$, most notably the Caporaso-Harris recursive algorithm [10]; see also [15. 19, 22, 41, 42]. The values $N_{d, g}$ for small $d$ and $g$ have been tabulated many times over (see ibid.); we do it again in Figure 1 .

\begin{tabular}{ccccccc}
\hline & $d=1$ & $d=2$ & $d=3$ & $d=4$ & $d=5$ & $d=6$ \\
\hline$N_{d, 0}$ & 1 & 1 & 12 & 620 & 87304 & 26312976 \\
$N_{d, 1}$ & 0 & 0 & 1 & 225 & 87192 & 57435240 \\
$N_{d, 2}$ & 0 & 0 & 0 & 27 & 36855 & 58444767 \\
$N_{d, 3}$ & 0 & 0 & 0 & 1 & 7915 & 34435125 \\
$N_{d, 4}$ & 0 & 0 & 0 & 0 & 882 & 12587820 \\
$N_{d, 5}$ & 0 & 0 & 0 & 0 & 48 & 2931600 \\
$N_{d, 6}$ & 0 & 0 & 0 & 0 & 1 & 437517 \\
\hline
\end{tabular}

Fig. 1. Gromov-Witten invariants $N_{d, g}$ for $d \leq 6$ and $g \leq 6$.

\begin{tabular}{ccccccc}
\hline & $d=1$ & $d=2$ & $d=3$ & $d=4$ & $d=5$ & $d=6$ \\
\hline$N^{d, 0}$ & 1 & 1 & 1 & 1 & 1 & 1 \\
$N^{d, 1}$ & 0 & 3 & 12 & 27 & 48 & 75 \\
$N^{d, 2}$ & 0 & 0 & 21 & 225 & 882 & 2370 \\
$N^{d, 3}$ & 0 & 0 & 15 & 675 & 7915 & 41310 \\
$N^{d, 4}$ & 0 & 0 & 0 & 666 & 36975 & 437517 \\
$N^{d, 5}$ & 0 & 0 & 0 & 378 & 90027 & 2931831 \\
$N^{d, 6}$ & 0 & 0 & 0 & 105 & 109781 & 12597900
\end{tabular}

Fig. 2. Severi degrees $N^{d, \delta}$ for $d \leq 6$ and $\delta \leq 6$. The numbers in italics include reducible curves.

Closely related to the numbers $N_{d, g}$ are the Severi degrees $N^{d, \delta}$, defined as follows: $N^{d, \delta}$ is the number of (possibly reducible) degree $d$ plane curves which have $\delta$ nodes and pass through a generic configuration of $d(d+3) / 2-\delta$ points on the plane. Figure 2 shows the values $N^{d, \delta}$ for small $d$ and $\delta$. 
For an irreducible plane curve of degree $d$, genus $g$, and $\delta$ double points, we have

$$
\delta+g=(d-1)(d-2) / 2
$$

for this reason, the number of nodes $\delta$ is called the cogenus of a curve (be it irreducible or not). It follows from Bezout's theorem that a degree $d$ nodal curve of cogenus $\delta \leq d-2$ must be irreducible; hence

$$
N^{d, \delta}=N_{d,(d-1)(d-2) / 2-\delta} \quad \text { for } d \geq \delta+2 .
$$

More generally, the Severi degrees can be recovered from the Gromov-Witten numbers by the following well known (and easy to justify) procedure. Fix a finite set $M$ of cardinality $d(d+3) / 2-\delta$; we can think of $M$ as an indexing set for a point configuration. Split $M$ into an unordered disjoint union of subsets $M=\bigcup_{j} M_{j}$; each such choice of a splitting corresponds to a distribution of the points in a configuration among the irreducible components of a curve. Then pick integers $d_{j}>0$ (to serve as degrees of those components) and $\delta_{j} \geq 0$ (their cogenera) so that the following natural conditions are satisfied:

$$
\begin{aligned}
& \sum_{j} d_{j}=d, \\
& \sum_{j} \delta_{j}+\sum_{\left\{j, j^{\prime}\right\}} d_{j} d_{j^{\prime}}=\delta
\end{aligned}
$$

(the second sum is over unordered pairs of distinct indices $j$ and $j^{\prime}$ ),

$$
\text { each } M_{j} \text { has cardinality }\left|M_{j}\right|=d_{j}\left(d_{j}+3\right) / 2-\delta_{j} \text {. }
$$

Using the notation $g_{j}=\left(d_{j}-1\right)\left(d_{j}-2\right) / 2-\delta_{j}$, we then have

$$
N^{d, \delta}=\sum_{M=\bigcup} \sum_{M_{j}} \prod_{\left(d_{j}, \delta_{j}\right)} N_{j} N_{j}, g_{j},
$$

the sum over all unordered splittings $M=\bigcup M_{j}$ and all choices of $d_{j}$ 's and $\delta_{j}$ 's satisfying (1.7)-1.9.

Example 1.8 (The Number of the Beast). The number of 4-nodal plane quartics through ten generic points is $N^{4,4}=666$, computed as follows. Direct inspection shows that there are precisely two kinds of splittings of a 10-element set $M$ that work for $d=\delta=4$ :

- $M=M_{1} \cup M_{2}$ with $\left|M_{1}\right|=2, d_{1}=1, \delta_{1}=0, g_{1}=0,\left|M_{2}\right|=8, d_{2}=3, \delta_{2}=1, g_{2}=0$ (a line through two points and a rational cubic through eight points);

- $M=M_{1} \cup M_{2}$ with $\left|M_{1}\right|=\left|M_{2}\right|=5, d_{1}=d_{2}=2, \delta_{1}=\delta_{2}=0, g_{1}=g_{2}=0$ (two conics, each passing through five points in a configuration).

This yields $N^{4,4}=\left(\begin{array}{c}10 \\ 2\end{array}\right) N_{1,0} N_{3,0}+\frac{1}{2}\left(\begin{array}{c}10 \\ 5\end{array}\right) N_{2,0}^{2}=45 \cdot 1 \cdot 12+\frac{1}{2} \cdot 252 \cdot 1^{2}=666$. 
Combining formula 1.10 with Theorem 1.6 we obtain the following combinatorial rule.

Corollary 1.9. The Severi degree $N^{d, \delta}$ is equal to

$$
N^{d, \delta}=\sum_{M=\bigcup M_{j}} \sum_{\left(d_{j}, \delta_{j}, \mathcal{D}_{j}\right)} \prod_{j} \mu\left(\mathcal{D}_{j}\right) v\left(\mathcal{D}_{j}\right),
$$

the sum over all unordered splittings $M=\bigcup M_{j}$ of the set $\{1, \ldots, d(d+3) / 2-\delta\}$, all choices of integers $d_{j}>0$ and $\delta_{j} \geq 0$ satisfying (1.7)-(1.9), and all collections of labeled floor diagrams $\mathcal{D}_{j}$, each of respective degree $d_{j}$ and genus $g_{j}=\left(d_{j}-1\right)\left(d_{j}-2\right) / 2-\delta_{j}$, and supported on the vertex set $M_{j}$.

To rephrase, the combinatorial rule for Severi degrees is the same as for the GromovWitten numbers $N_{d, g}$ except that one needs to drop the condition that the labeled floor diagrams involved be connected.

\section{Tropical curves}

Let us review the basic notions of tropical curves, both abstract and parametrized; see [21, 27, 28] for further details.

Throughout this section, $\bar{C}$ is a topological space homeomorphic to a compact onedimensional cell complex, i.e., a finite graph. We will assume that the underlying graph of $\bar{C}$ has no loops, no vertices of degree 2 , and at least one vertex of degree $\geq 3$ in each connected component.

Definition 2.1 (Valencies). A small neighborhood of a point $c \in \bar{C}$ is homeomorphic to a union of $k$ distinct rays in an affine space emanating from the same origin. We call $k$ the valency of $c$; accordingly, $c$ is called $k$-valent. All but finitely many points in $\bar{C}$ are 2 -valent. Let $C$ denote the subset of $\bar{C}$ obtained by removing all the (finitely many) points of valency 1 (the univalent vertices).

Definition 2.2 (Tropical curves). A tropical structure on $C$ is an inner complete metric on $C$. It can be described by specifying the lengths of all the edges of the underlying graph; these lengths are $+\infty$ for the edges incident to the removed univalent vertices (the unbounded edges), and are positive real numbers for the remaining (bounded) edges. A space $C$ as above endowed with a tropical structure is called a (nonparametrized, or abstract) tropical curve. Such a tropical curve is irreducible if $C$ is connected. We call the first Betti number of $C$ the genus of the tropical curve.

Example 2.3. Figure 3 a) shows a graph $\bar{C}$ with 12 univalent and 12 trivalent vertices, and with 12 bounded and 12 unbounded edges. Figure 3 b) shows an irreducible tropical curve of genus $g=1$ obtained from $\bar{C}$ by removing the univalent vertices and assigning the lengths $\ell_{1}, \ldots, \ell_{12}$ to its bounded edges. 


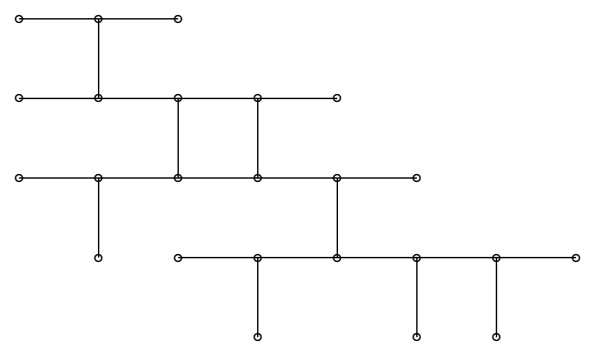

(a)

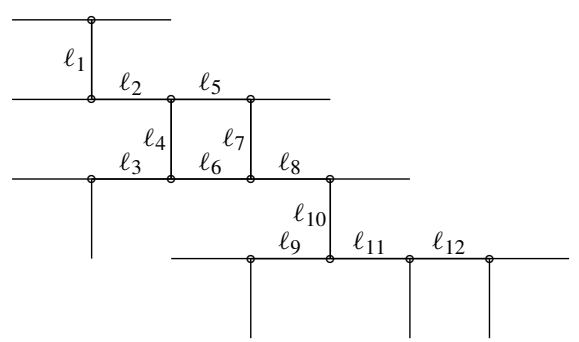

(b)

Fig. 3. A graph and a related tropical curve.

Definition 2.4 (Tropical morphisms and plane tropical curves). A map $h: C \rightarrow \mathbb{R}^{n}$ is called a tropical morphism if it satisfies the following properties:

- $h$ is affine along each edge $e$ in $C$. To be precise, let $a \in C$ be an endpoint of $e$ (thus $a$ is not univalent); then there is a vector $\Delta_{a}(e) \in \mathbb{R}^{n}$ such that the restriction of $h$ to $e$ is given by

$$
h(c)=h(a)+\ell(a, c) \Delta_{a}(e)
$$

here $\ell(a, c)$ denotes the length of the segment $[a, c]$ of the edge $e$.

- the vectors $\Delta_{a}(e)$ have integer coordinates;

- for a fixed vertex $a$, the vectors $\Delta_{a}(e)$ satisfy the balancing condition (cf. [28])

$$
\sum_{e} \Delta_{a}(e)=0
$$

where the sum is taken over all edges $e$ adjacent to $a$.

From now on we focus on the case $n=2$. A morphism from a tropical curve $C$ to $\mathbb{R}^{2}$ is called a (parametrized) plane tropical curve, or a tropical curve in $\mathbb{R}^{2}$.

Such a curve assigns positive integer weights to the edges in $C$, as follows. The weight $w(e)$ of an edge $e$ is the greatest common divisor of the coordinates of the integer vector $\Delta_{a}(e)$. In view of (2.2) below, this does not depend on the choice of $a$.

The sets $h(C) \subset \mathbb{R}^{2}$ obtained as the images of tropical morphisms were originally introduced by Aharony, Hanany, and Kol [1] under the name of $(p, q)$-webs.

Example 2.5 (cf. Example 2.3). Figure 4 shows the image of a particular morphism (a plane tropical curve) $h: C \rightarrow \mathbb{R}^{2}$ where $C$ is the tropical curve from Figure 3 . The weights of all edges are 1 except for one edge of weight 2 (the edge whose length is $\ell_{10}$ ). The integer vectors $\Delta_{a}(e)$ can be read off from the picture as follows: $\Delta_{a}(e)$ is $w(e)$ times the primitive vector of the segment or ray representing $e$, pointing away from the image 


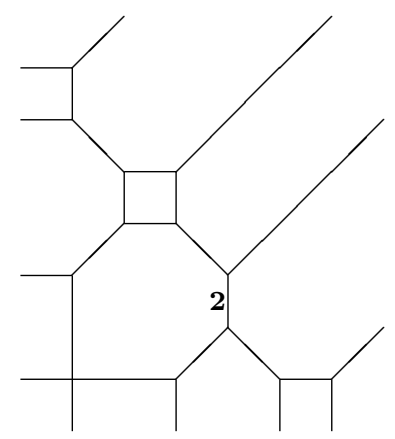

Fig. 4. The image of a plane tropical curve.

of $a$. Thus in this example, the values taken by $\Delta_{a}(e)$ are $(0, \pm 1),( \pm 1,0),( \pm 1, \pm 1)$, and $(0, \pm 2)$.

If $e$ is a bounded edge, i.e., an edge connecting two vertices $a$ and $b$ in $C$, then

$$
\Delta_{a}(e)+\Delta_{b}(e)=0 .
$$

If $e$ is unbounded, then it has a unique endpoint $a \in C$; consequently, the notation $\Delta(e)=$ $\Delta_{a}(e)$ is unambiguous. Let $E^{\circ}$ denote the set of all unbounded edges. It follows from 2.1. and (2.2) that

$$
\sum_{e \in E^{\circ}} \Delta(e)=0
$$

Definition 2.6 (Degree of a plane tropical curve). For a vector $v=(p, q) \in \mathbb{Z}^{2}$, set

$$
\langle v\rangle=\max (p, q, 0) .
$$

The (projective) degree of a plane tropical curve $h: C \rightarrow \mathbb{R}^{2}$ is defined by

$$
\operatorname{deg} h=\sum_{e \in E^{\circ}}\langle\Delta(e)\rangle .
$$

Thus the degree depends on the map $h$-unlike the genus, which only depends on the curve $C$.

To illustrate, the plane curve in Example 2.5 has degree $d=4$.

Remark 2.7. For all curves $h$ to be considered in this paper (say of degree $d$ ), the col-

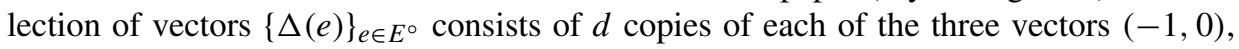
$(0,-1)$, and $(1,1)$, so that formula (2.3) yields $\operatorname{deg} h=d$. 


\section{The combinatorial correspondence theorems}

\subsection{Combinatorial rule for the ordinary Gromov-Witten invariants}

The correspondence theorem of Tropical Geometry [27] reduces the problems of counting plane complex curves with prescribed properties to the appropriate tropical versions of the same problems, that is, to (weighted) enumeration of certain plane tropical curves. In this section, we describe a setting that leads to a bijection between such tropical curves and some purely combinatorial objects, namely marked floor diagrams of Section 1. Combining the two constructions, we then obtain a "combinatorial correspondence theorem" that allows one to answer questions in enumerative geometry of the complex plane in direct combinatorial terms.

Definition 3.1 (Elevators). Let $h: C \rightarrow \mathbb{R}^{2}$ be a plane tropical curve. An edge $e$ in $C$ (either bounded or unbounded) is called an elevator of $h$ if the image $h(e) \subset \mathbb{R}^{2}$ is vertical, i.e., if the vector(s) $\Delta_{a}(e)$ are nonzero and parallel to $(0,1)$. The two possible orientations of an elevator $e$ are naturally called up and down.

Definition 3.2 (Floor diagram of a plane tropical curve). A floor of a plane tropical curve $h: C \rightarrow \mathbb{R}^{2}$ is a connected component (in $C$ ) of the union of all edges which are not elevators. The floor diagram of $h$ is an oriented weighted graph $\mathcal{D}(h)$ obtained from $C$ by removing (the interiors of) all unbounded edges, collapsing each floor to a single vertex, orienting all remaining edges (which correspond to bounded elevators) downwards, and keeping their weights. Thus, the vertices of $\mathcal{D}(h)$ correspond to the floors, and the edges to the bounded elevators (directed downwards).

Example 3.3 (cf. Examples 2.3 and 2.5). The plane tropical curve $h: C \rightarrow \mathbb{R}^{2}$ whose image is shown in Figure 4 has eight elevators: four bounded and four unbounded. They are precisely the eight vertical edges in Figure 3 (b), which are represented by the eight vertical segments and rays in Figure 3 (a).

The four floors of $h$ are formed by the horizontal edges of $C$ as shown in Figure 3 (b). The floor diagram $\mathcal{D}(h)$ is obtained by removing the unbounded edges, contracting each floor to a point, directing the four remaining edges downwards, and assigning weights $1,1,1,2$ to them. The result is a diagram isomorphic to the one shown in (1.3).

In general, a floor diagram of a plane tropical curve may not admit a labeling satisfying the conditions in Definition 1.1, so it might not correspond to any labeled floor diagram in the sense of that definition. In particular, the acyclicity condition and the divergence condition (1.1) cannot be guaranteed. It turns out however that the floor diagrams of plane curves passing through point configurations of certain kind always satisfy the requisite conditions, as we explain next.

Definition 3.4 (Vertically stretched configurations). A $(3 d-1+g)$-element set

$$
\mathcal{P}=\left\{\left(x_{1}, y_{1}\right),\left(x_{2}, y_{2}\right), \ldots,\left(x_{3 d-1+g}, y_{3 d-1+g}\right)\right\} \subset \mathbb{R}^{2}
$$


is called a vertically stretched $(d, g)$-configuration if

$$
\begin{gathered}
x_{1}<\cdots<x_{3 d-1+g}, \quad y_{1}<\cdots<y_{3 d-1+g}, \\
\min _{i \neq j}\left|y_{i}-y_{j}\right|>\left(d^{3}+d\right) \cdot \max _{i \neq j}\left|x_{i}-x_{j}\right| .
\end{gathered}
$$

Such a configuration $\mathcal{P}$ comes equipped with a "downward" linear order in which higher points precede the lower ones.

Remark 3.5. The conditions in Definition 3.4 can be relaxed by removing the second string of inequalities, changing the coefficient $d^{3}+d$, and/or removing the min and the max. We do not make an attempt to determine the weakest possible conditions that ensure the desired properties of associated tropical curves; instead, we chose the version that makes subsequent arguments as simple as possible.

We say that a plane tropical curve passes through a configuration $\mathcal{P} \subset \mathbb{R}^{2}$ if the image of the curve contains $\mathcal{P}$, i.e., $h(C) \supset \mathcal{P}$.

The following key lemma is a restatement of a result that can be extracted from 9 . Section 5].

Lemma 3.6. Let $\mathcal{P}$ be a vertically stretched $(d, g)$-configuration, and $h$ an irreducible plane tropical curve of degree $d$ and genus $g$ passing through $\mathcal{P}$. Then:

- each floor of $h$ contains a unique point in $\mathcal{P}$;

- the linear ordering of the floors induced from $\mathcal{P}$ makes $\mathcal{D}(h)$ a labeled floor diagram of degree $d$ and genus $g$ (cf. Definition 1.1p;

- each elevator of $h$ contains a unique point in $\mathcal{P}$;

- the linear ordering of the floors and elevators induced from $\mathcal{P}$ produces a marking of $\mathcal{D}(h)$, denoted by $\widetilde{\mathcal{D}}(h, \mathcal{P})$ (cf. Definition 1.4).

To amplify, such a curve $h$ has exactly $d$ floors, so that $\mathcal{D}(h)$ has $d$ vertices; each floor is contractible, so $\mathcal{D}(h)$, like $h$, has genus $g$; the graph $\mathcal{D}(h)$ is acyclic; and it satisfies the divergence condition.

Thus, each of the $d$ floors of $h$ is a graph of a continuous piecewise-linear function, with slopes at the left and right ends equal to 0 and 1, respectively. See Figure 5.

In fact, much more is true.

Theorem 3.7. Let $\mathcal{P}$ be a vertically stretched $(d, g)$-configuration. Then the mapping $h \mapsto \widetilde{\mathcal{D}}(h, \mathcal{P})$ establishes a bijective correspondence between the irreducible plane tropical curves of degree $d$ and genus $g$ passing through $\mathcal{P}$ and the marked floor diagrams of degree $d$ and genus $g$.

Proof. Let $\widetilde{\mathcal{D}}$ be a marking of a labeled floor diagram $\mathcal{D}$ of degree $d$ and genus $g$. We need to show that $\widetilde{\mathcal{D}}=\widetilde{\mathcal{D}}(h, \mathcal{P})$ for a unique plane tropical curve $h$ of the same degree and genus passing through $\mathcal{P}$. To do that, let us describe the structure of such a curve in concrete detail. Its construction from a given marked floor diagram $\widetilde{\mathcal{D}}$ and a point configuration $\mathcal{P}$ will then proceed by "reverse engineering". 


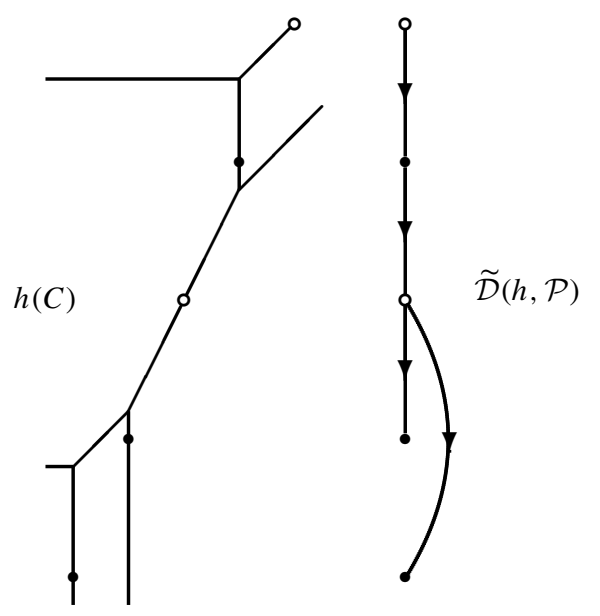

Fig. 5. Marked floor diagram of a tropical curve.

Since the vertex set of $\widetilde{\mathcal{D}}$ and the configuration $\mathcal{P}$ are linearly ordered sets of the same cardinality, there is a canonical order-preserving bijection $v \mapsto p(v)$ between them. It will be convenient to distinguish between "white" and "black" points $p(v) \in \mathcal{P}$, depending on whether $v$ comes from $\mathcal{D}$ or not. Each white vertex lies on the respective floor, and each black vertex lies on the corresponding elevator.

As we scan a floor $F_{v}$ passing through a white vertex $p(v)$ from its right end all the way to the left, the slope of the floor changes each time it meets an elevator. Specifically, the process unfolds as follows. The initial slope at the right end is 1 . An elevator $e_{u}$ arriving from above (respectively, from below) and passing through a black vertex $p(u)$ increases (respectively, decreases) the slope by $w\left(e_{u}\right)$. Due to the way the points in $\mathcal{P}$ are placed, the order in which those elevators hit the floor $F_{v}$ as we scan it right to left is precisely the (top-down) order in which the corresponding vertices $u$ (that is, all the black vertices connected to $v$ ) appear in $\widetilde{\mathcal{D}}$. Consequently, the slope of a segment $S_{u}$ of $F_{v}$ that is bounded on the right by an elevator $e_{u}$ is uniquely determined by the combinatorics of $\widetilde{\mathcal{D}}$ : it is equal to 1 plus a signed sum of weights of all edges in $\widetilde{\mathcal{D}}$ connecting $v$ to vertices $\leq u$; the edges arriving at $v$ contribute with a positive sign while the edges leaving $v$, with a negative one. Furthermore, the right endpoint of $S_{u}$ lies on the vertical line passing through $p(u)$ while the left endpoint lies on the vertical line passing through $p\left(u^{\prime}\right)$, where $u^{\prime}$ is the vertex in $\widetilde{\mathcal{D}}$ immediately following $u$ in the ordered list of vertices connected to $v$. To summarize, the combinatorics of $\widetilde{\mathcal{D}}$ determines the slopes of the segments making up the floor $F_{v}$, while the vertical lines passing through the points $p(u)$, for all $u$ connected to $v$, determine the $x$-coordinates of the breakpoints on $F_{v}$. This defines $F_{v}$ up to a vertical shift; the latter is determined by the condition that $p(v) \in F_{v}$.

The recipe for constructing a (necessarily unique) tropical curve $h$ with the desired properties is now clear: each floor $F_{v}$ is described by the above rule, and the vertical elevators are then drawn through the black points, bounded by the appropriate floors (or 
going all the way down in case of unbounded elevators). Since the slopes of floor segments cannot exceed $d$, condition (3.1) guarantees that each floor $F_{v}$ constructed in this way will fit below (respectively above) all black vertices $p(u)$ for $u \leq v$ (resp., $v \leq u$ ), ensuring that the recipe works. It is also clear that the curve $h$ constructed in this way will be irreducible, and will have the required genus and degree (for the latter, cf. Remark 2.7).

Theorem 3.7 is illustrated in Appendix B, which shows the nine tropical rational cubics passing through a vertically stretched $(3,0)$-configuration of eight points, alongside their respective marked floor diagrams.

Theorem 3.8 below is a special case of the (geometric) "correspondence theorem" [27, Theorem 1].

For a positive real number $t$, let $\log _{t}:\left(\mathbb{C}^{\times}\right)^{2} \rightarrow \mathbb{R}^{2}$ denote the map defined by

$$
\log _{t}(z, w)=\left(\log _{t}|z|, \log _{t}|w|\right) .
$$

Theorem 3.8. Let $\mathcal{P}$ be a vertically stretched $(d, g)$-configuration. Let $t$ be a sufficiently large positive number. Then for any configuration $\mathcal{P}^{\mathbb{C}} \subset\left(\mathbb{C}^{\times}\right)^{2}$ of $3 g-1+d$ points such that $\log _{t}(\mathcal{P} \mathbb{C})=\mathcal{P}$, there is a canonical surjective "tropicalization" map

$$
\gamma \mapsto \operatorname{Trop}_{\mathcal{P}^{\mathbb{C}}, t}(\gamma)
$$

from the set of irreducible complex algebraic curves $\gamma$ of degree $d$ and genus $g$ passing through $\mathcal{P}^{\mathbb{C}}$ to the set of irreducible plane tropical curves $h$ of degree $d$ and genus $g$ passing through $\mathcal{P}$. Under this map, the preimage $\operatorname{Trop}_{\mathcal{P}}^{-1}, t(h)$ of each such curve $h$ consists of $\mu(\mathcal{D}(h))$ distinct complex curves.

Proof. To adapt the correspondence theorem to our current setup, fix the Newton polygon of the curves under consideration to be the set

$$
\Delta_{d}=\{(i, j): i \geq 0, j \geq 0, i+j \leq d\},
$$

and observe that for a plane tropical curve $h$ passing through $\mathcal{P}$, the complex multiplicity $\mu_{\mathbb{C}}(h)$ (as defined in [27]) is equal to the multiplicity of the labeled floor diagram $\mathcal{D}(h)$ as defined in 1.2.

Combining Theorems 3.7 and 3.8 , we obtain the following enhancement of Theorem 1.6

Theorem 3.9 (Combinatorial correspondence theorem for plane curves). Let $\mathcal{P} \subset \mathbb{R}^{2}$ be a vertically stretched $(d, g)$-configuration. Let $t$ be sufficiently large. Let $\mathcal{P}^{\mathbb{C}} \subset\left(\mathbb{C}^{\times}\right)^{2}$ be a configuration of $3 g-1+d$ points such that $\log _{t}\left(\mathcal{P}^{\mathbb{C}}\right)=\mathcal{P}$. Then the composition

$$
\gamma \mapsto \widetilde{\mathcal{D}}\left(\operatorname{Trop}_{\mathcal{P}} \mathbb{C}_{, t}(\gamma), \mathcal{P}\right)
$$

is a surjection from the set of irreducible complex algebraic curves $\gamma$ of degree $d$ and genus g passing through $\mathcal{P} \mathbb{C}$ to the set of marked floor diagrams $\widetilde{\mathcal{D}}$ of degree $d$ and genus g. Under this map, the preimage of a marking $\widetilde{\mathcal{D}}$ of a labeled floor diagram $\mathcal{D}$ consists of $\mu(\mathcal{D})$ distinct complex curves. Consequently, 1.5 holds. 


\subsection{Combinatorial rule for the relative Gromov-Witten invariants}

In this section, we give a generalization of the combinatorial correspondence theorem (Theorem 3.9 to the problem of counting complex curves of given degree and genus which, in addition to passing through a given point configuration, satisfy some prescribed tangency conditions. This will require a suitable generalization of the notion of a marking of a labeled floor diagram.

The tangency conditions we will be working with are described by integer partitions. In dealing with the latter, we will use the standard notational conventions of the combinatorial theory of partitions (see, e.g., [37, Section 1.3] or [38, Section 7.2]) rather than those used by Caporaso and Harris [10] in their classical treatment of the subject. Recall that a partition $\lambda=\left(\lambda_{1}, \lambda_{2}, \ldots\right)$ of an integer $n \geq 0$ is a weakly decreasing sequence of nonnegative integers $\lambda_{i}$ whose sum is equal to $n$ :

$$
|\lambda|=\lambda_{1}+\lambda_{2}+\cdots=n .
$$

The nonzero $\lambda_{i}$ 's are called the parts of $\lambda$. The number of parts is the length of $\lambda$, denoted $\ell(\lambda)$. We write

$$
\lambda=\left\langle 1^{\alpha_{1}} 2^{\alpha_{2}} \cdots\right\rangle
$$

to express the fact that $\lambda$ has $\alpha_{i}$ parts equal to $i$, for each $i$. Thus $\ell(\lambda)=\alpha_{1}+\alpha_{2}+\cdots$.

Our first goal is to extend Theorem 3.8 to a more general setting. This will require some terminological preparation.

Definition 3.10 (Tangency conditions described by pairs of partitions). Let $\lambda$ and $\rho$ be two integer partitions. Let

$$
\mathcal{P}_{\lambda}=\left(p_{1}>\cdots>p_{\ell(\lambda)}\right)
$$

be a configuration of $\ell(\lambda)$ points in $\mathbb{R}$, and let

$$
\mathcal{P}_{\lambda}^{\mathbb{C}}=\left\{\left(x_{1}, 0\right), \ldots,\left(x_{\ell(\lambda)}, 0\right)\right\} \subset \mathbb{C}^{\times} \times \mathbb{C}
$$

be a configuration of points on the $x$-axis $\mathbb{C}^{\times} \times\{0\} \subset \mathbb{C}^{\times} \times \mathbb{C}$ such that $\log _{t}\left|x_{i}\right|=p_{i}$ for every $i$. We say that a complex curve $\gamma$ has $\left(\lambda, \mathcal{P}_{\lambda}^{\mathbb{C}}, \rho\right)$-tangency to the $x$-axis if $\gamma$ meets the latter at $\ell(\lambda)+\ell(\rho)$ points, as follows:

- $\gamma$ passes through $\mathcal{P}_{\lambda}^{\mathbb{C}}$, with tangency to the $x$-axis of degree $\lambda_{i}$ at each point $\left(x_{i}, 0\right)$;

- $\gamma$ passes through some other $\ell(\rho)$ points on the $x$-axis, with the degrees of tangency to the $x$-axis at those points forming the partition $\rho$.

Definition 3.11 (Grounding conditions for plane tropical curves). Let $h: C \rightarrow \mathbb{R}^{2}$ be a plane tropical curve of degree $d$ and genus $g$. A ground elevator of $h$ is an unbounded elevator $e$ (see Definition 3.1) for which the vector $\Delta(e)$ (see Definition 2.4) is a positive multiple of $(0,-1)$.

We say that a tropical curve $h: C \rightarrow \mathbb{R}^{2}$ is $\left(\lambda, \mathcal{P}_{\lambda}, \rho\right)$-grounded (cf. (3.4) ) if

- each point $\left(p_{i}, 0\right) \in \mathcal{P}_{\lambda} \times\{0\}$ is contained in a ground elevator of weight $\lambda_{i}$;

- the weights of the remaining ground elevators form the partition $\rho$.

See Figure 6 for an example. 


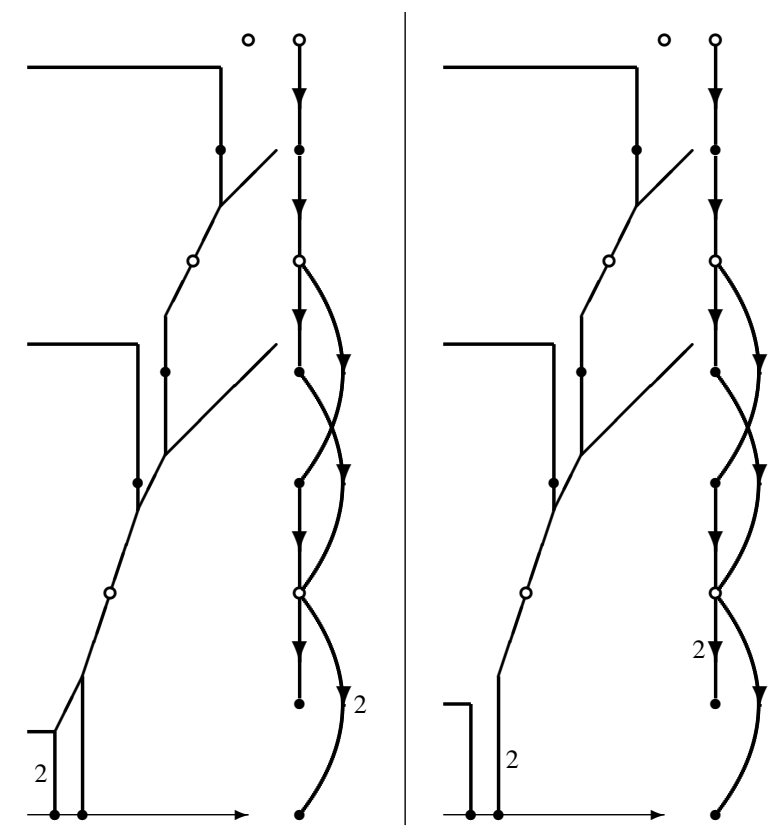

Fig. 6. Two tropical cubics passing through six vertically stretched points and $(\lambda, \mathcal{P} \lambda, \rho)$-grounded with $\lambda=\emptyset, \mathcal{P}=\emptyset$, and $\rho=(2,1)$. The multiplicity of each tropical cubic is 2 ; thus $N_{3,1}(\emptyset,(2,1))=4$.

We define the (complex) multiplicity of such a tropical curve as the number

$$
\mu_{\rho}(h)=\prod_{e} w^{2}(e) \prod_{i=1}^{\ell(\rho)} \rho_{i},
$$

where $e$ runs over all bounded elevators.

Theorem 3.12 below is a slight generalization of Theorem 3.8 (which is a special case of the correspondence theorem [27, Theorem 1]), and can be proved in a similar way. The proof will appear in [29].

Theorem 3.12. Let $\mathcal{P}$ be a generic configuration of $2 d-1+g+\ell(\rho)$ points in $\mathbb{R}^{2}$ satisfying the condition 3.1. Let $\mathcal{P}^{\mathbb{C}} \subset\left(\mathbb{C}^{\times}\right)^{2}$ be a configuration of points such that $\log _{t}\left(\mathcal{P}^{\mathbb{C}}\right)=\mathcal{P}$. Let configurations $\mathcal{P}_{\lambda}$ and $\mathcal{P}_{\lambda}^{\mathbb{C}}$ be as in Definition 3.10. Let $t \in \mathbb{R}_{>0}$ be sufficiently large.

Then there is a canonical surjection from the set of irreducible complex algebraic curves of degree $d$ and genus $g$ passing through $\mathcal{P} \mathbb{C}$ and having $\left(\lambda, \mathcal{P}_{\lambda}^{\mathbb{C}}, \rho\right)$-tangency to the $x$-axis to the set of irreducible $\left(\lambda, \mathcal{P}_{\lambda}, \rho\right)$-grounded plane tropical curves of degree $d$ and genus $g$ passing through $\mathcal{P}$. Under this surjection, the preimage of each such tropical curve $h$ consists of $\mu_{\rho}(h)$ distinct complex curves. 
We generalize Definition 1.4, as follows. (A similar definition, with $g=0$, appeared in [2, Definition 4.2].)

Definition 3.13 (Floor diagrams marked by pairs of partitions). Let $\mathcal{D}$ be a labeled floor diagram of degree $d$ and genus $g$. Let $\lambda$ and $\rho$ be two partitions with $|\lambda|+|\rho|=d$. A $(\lambda, \rho)$-marking of $\mathcal{D}$ is a combinatorial decoration of $\mathcal{D}$ obtained by the following modification of the procedure used in Definition 1.4. We will illustrate the steps of this procedure using a running example, in which $d=4, g=1, \lambda=(2), \rho=(1,1)$, and $\mathcal{D}$ is the labeled floor diagram from Example 1.3 .

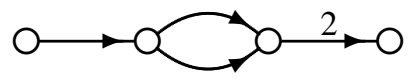

Step 0. Introduce $\ell(\lambda)$ new vertices denoted $v_{1}, \ldots, v_{\ell(\lambda)}$ :

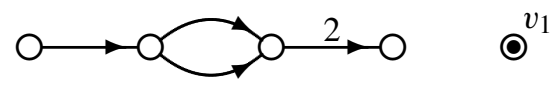

Step 1. For each original vertex $v$ in $\mathcal{D}$, introduce some number of additional new vertices (possibly none), and connect $v$ to each of them by a single edge directed away from $v$. In addition, introduce some edges (possibly none) directed from $v$ to $v_{1}, \ldots, v_{\ell(\lambda)}$. Assign positive integer weights to all these new edges so that

- for each original vertex $v$ in $\mathcal{D}$, the total weight of all new edges (of both kinds) originating at $v$ is equal to $1-\operatorname{div}(v)$;

- the weights of all edges arriving at $v_{1}, \ldots, v_{\ell(\lambda)}$ form the partition $\lambda$;

- the weights of all other new edges form the partition $\rho$.

Thus, the total weight of all new edges is equal to $\sum_{v}(1-\operatorname{div}(v))=d=|\lambda|+|\rho|$.

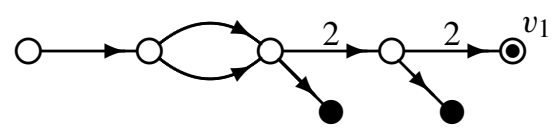

Step 2. Split each original edge $e$ of $\mathcal{D}$ in two, by inserting an extra vertex in the middle of $e$; the resulting two edges inherit $e$ 's orientation and weight.

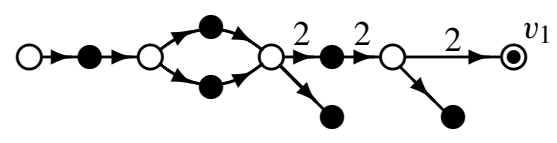

Let $\widetilde{G}=(\widetilde{V}, \widetilde{E})$ denote the acyclic directed graph obtained after Steps 1-2.

Step 3. Extend the linear ordering on the vertices of $\mathcal{D}$ to the set $\widetilde{V}$ so that, as before, each edge in $\widetilde{E}$ is directed from a smaller to a larger vertex. We also require $v_{1}$ to be the maximal element under the linear ordering, $v_{2}$ the second largest, etc.:

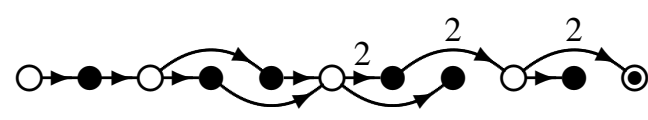


The resulting object $\widetilde{\mathcal{D}}$ is called a $(\lambda, \rho)$-marked floor diagram (of degree $d$ and genus $g$ ), or a $(\lambda, \rho)$-marking of the original labeled floor diagram $\mathcal{D}$. It is easy to see that $\widetilde{\mathcal{D}}$ has $2 d+g-1+\ell(\lambda)+\ell(\rho)$ vertices and $2 d+2 g-2+\ell(\lambda)+\ell(\rho)$ edges.

The number of distinct $(\lambda, \rho)$-markings of $\mathcal{D}$ is denoted by $v_{\lambda, \rho}(\mathcal{D})$. We also denote (cf. (3.5))

$$
\mu_{\rho}(\widetilde{\mathcal{D}})=\mu_{\rho}(\mathcal{D})=\mu(\mathcal{D}) \prod_{i=1}^{\ell(\rho)} \rho_{i}
$$

Remark 3.14. Let $\lambda=\emptyset$ and $\rho=\left\langle 1^{d}\right\rangle$. In this special case, we recover the ordinary notion of a marked floor diagram introduced in Definition 1.4 . We also recover $v_{\emptyset,\left\langle 1^{d}\right\rangle}(\mathcal{D})=v(\mathcal{D})$ and $\mu_{\emptyset,\left\langle 1^{d}\right\rangle}(\mathcal{D})=\mu(\mathcal{D})$.

More generally, let $\lambda=\left\langle 1^{k}\right\rangle$ and $\rho=\left\langle 1^{d-k}\right\rangle$. Then a $(\lambda, \rho)$-marked floor diagram is nothing but an ordinary marked floor diagram whose last $k$ vertices are sinks.

Definition 3.15 (Relative Gromov-Witten invariants). Let $N_{d, g}(\lambda, \rho)$ denote the number of irreducible complex algebraic curves of degree $d$ and genus $g$ passing through a generic configuration of $2 d-1+g+\ell(\rho)$ points in $\mathbb{C}^{2}$ and having $\left(\lambda, \mathcal{P}_{\lambda}^{\mathbb{C}}, \rho\right)$-tangency to the $x$-axis (see Definition 3.10 , for a given generic collection $\mathcal{P}_{\lambda}^{\mathbb{C}}$ of $\ell(\lambda)$ points on $\mathbb{C} \times\{0\}$.

Thus $N_{d, g}(\lambda, \rho)$ counts irreducible plane curves $\gamma$ of given degree and genus intersecting a given line $L$ (say the $x$-axis) with multiplicities described by $\lambda$ at a given collection of points on $L$, and with multiplicities described by $\rho$ at some other unspecified points on $L$; in addition, $\gamma$ must pass through a generic configuration of (appropriately many) points on the plane.

We note that the numbers $N_{d, g}(\lambda, \rho)$ are different from the numbers $N^{d, \delta}(\alpha, \beta)$ studied by Caporaso and Harris [10] since $N_{d, g}(\lambda, \rho)$ only counts irreducible curves. The Caporaso-Harris numbers are the generalizations of the Severi degrees discussed in Section 1.2, and can be similarly expressed as sums of the numbers $N_{d, g}(\lambda, \rho)$ (with positive integer coefficients) by considering possible partitions of a given configuration into subconfigurations lying on the irreducible components of a curve. Conversely, the numbers $N_{d, g}(\lambda, \rho)$ can be computed from the Caporaso-Harris numbers (also known as generalized Severi degrees) by an appropriate inclusion-exclusion procedure.

Remark 3.16. Let $\lambda$ be a partition with $|\lambda| \leq d$, and let $\rho=\left\langle 1^{d-|\lambda|}\right\rangle$. Then the tangency conditions associated with $\rho$ become vacuous. Consequently, the relative GromovWitten invariant $N_{d, g}\left(\lambda,\left\langle 1^{d-|\lambda|}\right\rangle\right)$ counts irreducible plane curves of degree $d$ and genus $g$ passing through a generic configuration of $\ell(\lambda)$ points on a given line with tangencies to the line described by the partition $\lambda$, and also passing through a generic configuration of $3 d-1+g-|\lambda|$ points on the plane.

In particular, the number $N_{d, g}\left(\left\langle 1^{k}\right\rangle,\left\langle 1^{d-k}\right\rangle\right)$ counts irreducible curves of degree $d$ and genus $g$ passing through a configuration of $3 d-1+g$ points on the plane that contains $k$ 
collinear points and is otherwise generic. For $k \leq 2$, we obviously get

$$
N_{d, g}\left(\emptyset,\left\langle 1^{d}\right\rangle\right)=N_{d, g}\left((1),\left\langle 1^{d-1}\right\rangle\right)=N_{d, g}\left((1,1),\left\langle 1^{d-2}\right\rangle\right)=N_{d, g} .
$$

At this point, the following analogue of Theorem 3.7 should come as no surprise. (A proof can be given along similar lines.)

Theorem 3.17. Under the assumptions of Theorem 3.12 there is a multiplicity-preserving bijection $h \mapsto \widetilde{\mathcal{D}}$ (that is, $\mu_{\rho}(h)=\mu_{\rho}(\widetilde{\mathcal{D}})$ ) between the plane tropical curves $h$ described in that theorem and the $(\lambda, \rho)$-marked floor diagrams of degree $d$ and genus $g$.

Theorems 3.12 and 3.17 imply the following generalization of Theorem 1.6 . (The case $g=0$ has been stated in [2, Theorem 4.4].)

Theorem 3.18. The relative Gromov-Witten invariant $N_{d, g}(\lambda, \rho)$ is given by

$$
N_{d, g}(\lambda, \rho)=\sum_{\mathcal{D}} \mu_{\rho}(\mathcal{D}) \nu_{\lambda, \rho}(\mathcal{D}),
$$

the sum over all labeled floor diagrams $\mathcal{D}$ of degree $d$ and genus $g$.

In other words, the number $N_{d, g}(\lambda, \rho)$ can be computed by counting $(\lambda, \rho)$-marked (labeled) floor diagrams $\widetilde{\mathcal{D}}$, each taken with multiplicity $\mu_{\rho}(\widetilde{\mathcal{D}})$.

\section{Computing relative Gromov-Witten invariants}

This section is devoted to applications of the combinatorial rule of Theorem 3.18

\subsection{Conics and cubics}

As a warm-up, let us look at the cases $d=2$ and $d=3$. (All of these enumerative invariants have been known since the 19th century.)

Example 4.1 (Plane conics). For $d=2$ and $g=0$, there are very few possibilities. By (3.7), we have

$$
N_{2,0}(\emptyset,(1,1))=N_{2,0}((1),(1))=N_{2,0}((1,1), \emptyset)=N_{2,0}=1,
$$

the unique plane conic through five generic points. This corresponds to the unique $(\lambda, \rho)$ marked floor diagram of multiplicity 1 . In the cases $(\lambda, \rho)=((2), \emptyset)$ and $(\lambda, \rho)=$ $(\emptyset,(2))$, the diagram is unique as well; the multiplicities are 1 and 2 , respectively, so $N_{2,0}((2), \emptyset)=1$ and $N_{2,0}(\emptyset,(2))=2$. This accounts for:

- the unique plane conic passing through three generic points and tangent to a given line at a given point;

- two plane conics passing through four given points and tangent to a given line. 
Example 4.2 (Elliptic plane cubics). For $d=3$ and $g=1$, there is only one labeled floor diagram, so combinatorial calculations are very simple. Applying Theorem 3.18 , we see that all relative Gromov-Witten invariants $N_{3,1}(\lambda, \rho)$ are equal to 1 , except for:

- $N_{3,1}((1),(2))=2$ (plane cubics passing through seven generic points and having two distinct common points with a given line, namely a given intersection point and an unspecified point of tangency);

- $N_{3,1}(\emptyset,(2,1))=4$ (plane cubics passing through eight generic points and tangent to a given line; cf. Figure 6;

- $N_{3,1}(\emptyset,(3))=3$ (plane cubics passing through seven generic points and having an unspecified point of order-3 tangency to a given line).

Example 4.3 (Rational plane cubics). There are three labeled floor diagrams $\mathcal{D}$ of degree $d=3$ and genus $g=0$. By 3.7), we have

$$
N_{3,0}(\emptyset,(1,1,1))=N_{3,0}((1),(1,1))=N_{3,0}((1,1),(1))=N_{3,0}=12
$$

(12 rational plane cubics through eight generic points); the combinatorial calculation is the same as in Example 1.7 The remaining cases are presented in Figure 7 For each labeled floor diagram $\mathcal{D}$ and each pair of partitions $(\lambda, \rho)$, the table shows the corresponding contribution $\mu_{\rho}(\mathcal{D}) \cdot v_{\lambda, \rho}(\mathcal{D})$ to the right-hand side of $(3.8)$. These contributions are then added together to get $N_{\lambda, \rho}$. For example, there are $N_{3,0}(\emptyset,(3))=21$ rational cubics passing through six generic points and having tangency of order 3 to a given line (at an unspecified point of inflection).

\begin{tabular}{cccccccc}
$\lambda=\emptyset$ & $\begin{array}{c}\lambda=\emptyset \\
\rho=(2,1)\end{array}$ & $\begin{array}{c}\lambda=(1) \\
\rho=(3)\end{array}$ & $\begin{array}{c}\lambda=(2) \\
\rho=(2)\end{array}$ & $\begin{array}{c}\lambda=\left\langle 1^{3}\right\rangle \\
\rho=\emptyset\end{array}$ & $\begin{array}{c}\lambda=(2,1) \\
\rho=\emptyset\end{array}$ & $\begin{array}{c}\lambda=(3) \\
\rho=\emptyset\end{array}$ \\
\hline 2 & $2 \cdot 4$ & $3 \cdot 0$ & $2 \cdot 1$ & $1 \cdot 3$ & $1 \cdot 3$ & $1 \cdot 1$ & $1 \cdot 0$ \\
\hline & $8 \cdot 2$ & $12 \cdot 1$ & $8 \cdot 1$ & $4 \cdot 1$ & $4 \cdot 1$ & $4 \cdot 1$ & $4 \cdot 1$ \\
\hline
\end{tabular}

Fig. 7. Combinatorial computation of the numbers $N_{3,0}(\lambda, \rho)$.

\subsection{Nonsingular and uninodal curves}

Nonsingular (or generic) plane algebraic curves have the maximal possible genus among all curves of degree $d$, namely

$$
g_{\max }=g_{\max }(d)=(d-1)(d-2) / 2 .
$$

There is only one labeled floor diagram $\mathcal{D}$ of degree $d$ and genus $g_{\max }$, namely one that looks like this (shown for $d=6$ and $g_{\max }=10$ ):

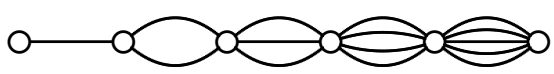


For this floor diagram, we have $\mu(\mathcal{D})=v(\mathcal{D})=1$, implying $N_{d, g_{\max }}=1$ (cf. (5.1)). For a pair of partitions $\lambda$ and $\rho$ with $|\lambda|+|\rho|=d$, the number of markings $\nu_{\lambda, \rho}(\mathcal{D})$ is equal to the number $o(\rho)$ of distinct permutations of the parts of $\rho$. That is, if

$$
\rho=\left\langle 1^{\beta_{1}} 2^{\beta_{2}} \cdots\right\rangle
$$

(cf. (3.3), then

$$
\nu_{\lambda, \rho}(\mathcal{D})=o(\rho)=\frac{(\ell(\rho)) !}{\beta_{1} ! \beta_{2} ! \cdots} .
$$

Combining (4.3) with 3.6 and 3.8 , we obtain the following formula.

Corollary 4.4. Let $\lambda$ and $\rho$ be partitions such that $|\lambda|+|\rho|=d$. Then the number of irreducible plane complex algebraic curves of degree $d$ passing through a generic configuration of $d(d+1) / 2+\ell(\rho)$ points and having tangencies to a given line described by $\lambda$ and $\rho$ (that is, $\lambda$ describes tangencies at given points whereas $\rho$ describes tangencies at unspecified points) is equal to

$$
N_{d, g_{\max }}(\lambda, \rho)=\rho_{1} \rho_{2} \cdots \frac{(\ell(\rho)) !}{\beta_{1} ! \beta_{2} ! \cdots} .
$$

Note that this number does not depend on $\lambda$.

Let us now turn to counting uninodal curves, i.e., those of genus

$$
g=g_{\max }-1=d(d-3) / 2 \quad(d \geq 3) .
$$

It is easy to see that there are $2 d-3$ labeled floor diagrams of degree $d$ and genus $g_{\max }-1$ (cf. [9, Proposition 6.1]); the five diagrams for $d=4$ and $g=2$ are shown in the second table of Appendix A. More specifically, there are $d-1$ diagrams $\mathcal{D}_{1}, \ldots, \mathcal{D}_{d-1}$ with $\mu\left(\mathcal{D}_{i}\right)=1$ and $\nu\left(\mathcal{D}_{i}\right)=2 i+1$; and $d-2$ additional diagrams $\mathcal{D}_{1}^{\prime}, \ldots, \mathcal{D}_{d-2}^{\prime}$ with $\mu\left(\mathcal{D}_{i}^{\prime}\right)=4$ and $\nu\left(\mathcal{D}_{i}^{\prime}\right)=i$. This gives

$N_{d, g_{\max }-1}=(3+5+\cdots+(2 d-1))+4(1+2+\cdots+(d-2))=3(d-1)^{2} ;$

cf. 5.2).

We next generalize the formula (4.5) to the setting involving tangency conditions.

Corollary 4.5. Let $\lambda=\left\langle 1^{\alpha_{1}} 2^{\alpha_{2}} \cdots\right\rangle$ and $\rho=\left\langle 1^{\beta_{1}} 2^{\beta_{2}} \cdots\right\rangle$ be partitions such that $|\lambda|+$ $|\rho|=d$. Then the number of uninodal irreducible plane complex algebraic curves of degree $d$ passing through a generic configuration of $(d-1)(d+2) / 2+\ell(\rho)$ points and having tangencies to a given line described by $\lambda$ and $\rho$ is given by the formula

$$
\begin{aligned}
& N_{d, g_{\max }-1}(\lambda, \rho) \\
& \quad= \begin{cases}\left((d-2)(3 d-2)+\alpha_{1}+\beta_{1}+(d-1) \frac{\beta_{1}}{\ell(\rho)}\right) N_{d, g_{\max }}(\lambda, \rho) & \text { if } \rho \neq \emptyset ; \\
(d-2)(3 d-2)+\alpha_{1} & \text { if } \rho=\emptyset .\end{cases}
\end{aligned}
$$

(Recall that $N_{d, g_{\max }}(\lambda, \rho)$ is given by 4.4).) 
Proof. We need to compute the quantities $\mu_{\rho}$ and $\nu_{\lambda, \rho}$ for each of the diagrams $\mathcal{D}_{i}$ and $\mathcal{D}_{i}^{\prime}$, then use (3.8). First, a little preparation. If $\beta_{1} \geq 1$, then denote

$$
\bar{\rho}=\left\langle 1^{\beta_{1}-1} 2^{\beta_{2}} 3^{\beta_{3}} \cdots\right\rangle,
$$

so that (cf. 4.3)

$$
o(\bar{\rho})=\frac{(\ell(\rho)-1) !}{\left(\beta_{1}-1\right) ! \beta_{2} ! \cdots}=\frac{\beta_{1}}{\ell(\rho)} o(\rho) .
$$

Now, calculations give (cf. 4.3):

$$
\begin{aligned}
\mu_{\rho}\left(\mathcal{D}_{i}\right) & =\rho_{1} \rho_{2} \cdots, \\
\mu_{\rho}\left(\mathcal{D}_{i}^{\prime}\right) & =4 \rho_{1} \rho_{2} \cdots, \\
\nu_{\lambda, \rho}\left(\mathcal{D}_{i}\right) & =(2 i+1) o(\rho) \quad(i \leq d-2), \\
\nu_{\lambda, \rho}\left(\mathcal{D}_{d-1}\right) & =(d-1) \frac{\beta_{1}}{\ell(\rho)} o(\rho)+\left(\alpha_{1}+\beta_{1}\right) o(\rho), \\
\nu_{\lambda, \rho}\left(\mathcal{D}_{i}^{\prime}\right) & =i o(\rho) \quad(i \leq d-2) .
\end{aligned}
$$

Collecting everything, we get

$$
\begin{aligned}
N_{d, g_{\max }-1}(\lambda, \rho) & =\rho_{1} \rho_{2} \cdots o(\rho)\left(\sum_{i=1}^{d-2}(6 i+1)+(d-1) \frac{\beta_{1}}{\ell(\rho)}+\alpha_{1}+\beta_{1}\right) \\
& =N_{d, g_{\max }}(\lambda, \rho)\left(3 d^{2}-8 d+4+(d-1) \frac{\beta_{1}}{\ell(\rho)}+\alpha_{1}+\beta_{1}\right) .
\end{aligned}
$$

\subsection{Curves passing through collinear points}

Let us next consider the cases $(\lambda, \rho)=\left(\left\langle 1^{k}\right\rangle,\left\langle 1^{d-k}\right\rangle\right)$ discussed in Remarks 3.14 and 3.16 Combining the latter with Theorem 3.18 , we obtain the following corollary.

Corollary 4.6. The number $N_{d, g}\left(\left\langle 1^{k}\right\rangle,\left\langle 1^{d-k}\right\rangle\right)$ of irreducible plane complex algebraic curves of degree $d$ and genus $g$ passing through a generic configuration of $3 d+g-k-1$ points and a generic configuration of $k$ collinear points is equal to $\sum_{\widetilde{\mathcal{D}}} \mu(\widetilde{\mathcal{D}})$, the sum over marked floor diagrams $\widetilde{\mathcal{D}}$ of degree $d$ and genus $g$ whose last $k$ vertices are sinks.

The special cases $k=0,1,2$ of Corollary 4.6 yield the ordinary Gromov-Witten numbers (cf. 3.7). Let us examine the case $k=3$.

Corollary 4.7. The number of irreducible plane complex algebraic curves of degree $d$ and genus $g$ passing through a generic configuration of $3 d+g-4$ points and through a generic triple of collinear points is given by the formula

$$
N_{d, g}\left(\left\langle 1^{3}\right\rangle,\left\langle 1^{d-3}\right\rangle\right)=N_{d, g}-(d-1) N_{d-1, g} .
$$


For example, there are $N_{3,0}\left(\left\langle 1^{3}\right\rangle, \emptyset\right)=N_{3,0}-2 N_{2,0}=10$ irreducible plane rational cubics passing through five generic points and three generic collinear points-matching the value in Figure 7

Proof of Corollary 4.7. Apply Corollary 4.6 with $k=3$. Note that in every (ordinary) marked floor diagram, the last two vertices are sinks. Hence $N_{d, g}\left(\left\langle 1^{3}\right\rangle,\left\langle 1^{d-3}\right\rangle\right)$ is equal to $N_{d, g}$ minus $\sum_{\widetilde{\mathcal{D}}} \mu(\widetilde{\mathcal{D}})$, the sum over marked floor diagrams $\widetilde{\mathcal{D}}$ of degree $d$ and genus $g$ in which the 3rd largest vertex $v$ is not a sink. Such a diagram $\widetilde{\mathcal{D}}$ looks like this (we only draw the edges of interest to us):

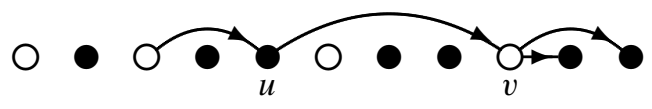

(Since there are two edges pointing away from $v$, there must be a unique edge $u \rightarrow v$ arriving at $v$, by the divergence condition.) Removing the three edges incident to $v$ creates a marked floor diagram $\widetilde{\mathcal{D}}^{\prime}$ of degree $d-1$ and genus $g$, with a distinguished sink $u$. Conversely, given such a marked diagram $\widetilde{\mathcal{D}}^{\prime}$ with a sink $u$ in it, $\widetilde{\mathcal{D}}$ is uniquely recovered. Note that each $\widetilde{\mathcal{D}}^{\prime}$ has $d-1$ sinks. Furthermore, $\mu\left(\widetilde{\mathcal{D}}^{\prime}\right)=\mu(\mathcal{D})$, and the claim follows.

\subsection{Curves with maximal tangency to a given line}

Let us now look at the problem of counting irreducible plane curves of degree $d$ and genus $g$ passing through appropriately many points and having maximal tangency (of order $d$ ) to a given line. The corresponding relative Gromov-Witten invariants come in two flavors, depending on whether the point of tangency is prescribed or not:

- $N_{d, g}((d), \emptyset)$ is the number of irreducible plane curves of degree $d$ and genus $g$ which pass through a generic configuration of $2 d+g-1$ points and have tangency of order $d$ to a given line $L$ at a given point $x \in L$;

- $N_{d, g}(\emptyset,(d))$ is the number of irreducible plane curves of degree $d$ and genus $g$ which pass through a generic configuration of $2 d+g$ points and have tangency of order $d$ to a given line $L$ at some point $x \in L$.

One surprising corollary of Theorem 3.18 is that these two numbers are related to each other in a very simple way.

Corollary 4.8. We have $N_{d, g}(\emptyset,(d))=d \cdot N_{d, g}((d), \emptyset)$.

Proof. It is easy to see that the corresponding two sets of $(\lambda, \rho)$-marked floor diagrams are the same whereas their multiplicities $\mu_{\rho}$ differ by a factor of $d$.

As we learned from R. Vakil, Corollary 4.8 can be seen to be a particular case of the Caporaso-Harris formula.

In the special case $g=0$ (counting irreducible plane rational curves maximally tangent to a given line), the relevant problem of combinatorial enumeration can be solved completely. As a result, we obtain a recurrence (see Theorem 4.9 below) that can be used to calculate as many numbers $N_{d, 0}((d), \emptyset)$ and $N_{d, 0}(\emptyset,(d))$ as one's computing resources allow. See Figure 8 . 


\begin{tabular}{rrr}
\hline$d$ & $N_{d, 0}((d), \emptyset)$ & $N_{d, 0}(\emptyset,(d))$ \\
\hline 1 & 1 & 1 \\
2 & 1 & 2 \\
3 & 7 & 21 \\
4 & 138 & 552 \\
5 & 5477 & 27385 \\
6 & 367640 & 2205840 \\
7 & 37541883 & 262793181 \\
8 & 5432772352 & 43462178816 \\
9 & 1059075055273 & 9531675497457 \\
10 & 267757626501504 & 2677576265015040 \\
11 & 85244466165571535 & 937689127821286885 \\
12 & 33379687015338236672 & 400556244184058840064 \\
13 & 15770655073870516443597 & 205018515960316713766761 \\
14 & 8847780392111931116474368 & 123868925489567035630641152 \\
15 & 5815426547948880787678282627 & 87231398219233211815174239405 \\
16 & 4426738320076692932937846865920 & 70827813121227086927005549854720 \\
\hline
\end{tabular}

Fig. 8. Number of irreducible rational curves maximally tangent to a line.

Theorem 4.9. The numbers $z(d)=N_{d, 0}((d), \emptyset)$ satisfy the recurrence relation

$$
z(d+1)=\sum_{k=1}^{d} \frac{(2 d) !}{k !} \sum_{\substack{a_{1}+\cdots+a_{k}=d \\ a_{1}, \ldots, a_{k}>0}} \prod_{i=1}^{k} \frac{a_{i}^{2} z\left(a_{i}\right)}{\left(2 a_{i}\right) !} .
$$

Proof. In order for a labeled floor diagram $\mathcal{D}$ to allow for a marking containing an edge of weight $d$ (necessarily pointing from the last vertex $v_{\text {last }}$ to the unique sink $v_{1}$ ), two conditions must be satisfied at each vertex $v$ in $\mathcal{D}$ :

- there is exactly one outgoing edge emanating from $v$;

- the inequality (1.1) holds with an equality sign.

These conditions mean that the labeled floor diagrams $\mathcal{D}$ under consideration can be identified with increasing rooted trees on the vertex set $\{1, \ldots, d\}$, i.e., with the labeled trees on $d$ vertices in which the labels increase along each simple path ending at the root vertex $d=v_{\text {last }}$. (To be literally precise, such trees are decreasing in the terminology of [37, 38], but this term would be misleading since we orient the edges towards the root rather than away from it.) It is immediate from the definitions that $\mu_{\emptyset}(\mathcal{D})=\mu(\mathcal{D})$ and $v_{(d), \emptyset}(\mathcal{D})=v(\mathcal{D})$, so we have $z(d)=N_{d, 0}((d), \emptyset)=\sum_{\mathcal{D}} \mu(\mathcal{D}) v(\mathcal{D})$, the sum over all increasing trees $\mathcal{D}$ on $d$ vertices. For example, referring to the first table in Appendix A, $z(3)=4 \cdot 1+1 \cdot 3=7$ and $z(4)=36 \cdot 1+9 \cdot 3+4 \cdot 3+4 \cdot 7+4 \cdot 5+1 \cdot 15=138$.

The hooklength $h(v)$ of a vertex $v$ in $\mathcal{D}$ (cf. [34, 3.12.18]) is, by definition, the number of vertices $u$ which precede $v$ in $\mathcal{D}$ (including $u=v$ ). It is easy to see from the divergence condition that the hooklengths of the non-root vertices are precisely the edge weights of $\mathcal{D}$. Thus $\mu(\mathcal{D})=\prod_{v \neq v_{\text {last }}}(h(v))^{2}$.

Consistent with the above, let a marked increasing tree on $2 d$ vertices be an increasing (rooted) tree $\widetilde{\mathcal{D}}$ obtained from an increasing tree $\mathcal{D}$ on $d$ vertices by appending an extra 
root vertex beyond the old one, introducing an extra vertex at the middle of each edge of $\mathcal{D}$, and extending the linear ordering to the resulting tree. We then have

$$
z(d)=\sum_{\widetilde{\mathcal{D}}} \prod_{v \neq v_{\text {last }}}(h(v))^{2},
$$

where the sum is over all marked increasing trees $\widetilde{\mathcal{D}}$ on $2 d$ vertices, and the product is over all non-root vertices of the corresponding tree $\mathcal{D}$.

The recurrence (4.6) can now be obtained using standard techniques of combinatorial enumeration (cf., e.g., [38, Chapter 5] or [5, Section 5.2]). A marked increasing tree $\widetilde{\mathcal{D}}$ on $2(d+1)$ vertices is uniquely decomposed, by cutting off the last two vertices (the old root and the new one) together with the edges incident to them, into a shuffle of some number $k$ of marked rooted trees $\widetilde{\mathcal{D}}_{1}, \ldots, \widetilde{\mathcal{D}}_{k}$ on $2 a_{1}, \ldots, 2 a_{k}$ vertices, respectively, where $\sum_{i} a_{i}=d$, and we numbered the subtrees arbitrarily by the integers $1, \ldots, k$. (To compensate for this additional choice, we will need to divide by $k$ ! at the end.) The multiset of non-root hooklengths of $\widetilde{\mathcal{D}}$ is the disjoint union of the multisets of hooklengths of $\widetilde{\mathcal{D}}_{1}, \ldots, \widetilde{\mathcal{D}}_{k}$ (including the root hooklengths $\left.a_{1}, \ldots, a_{k}\right)$. Hence $\mu(\widetilde{\mathcal{D}})=\prod_{i} \mu\left(\widetilde{\mathcal{D}}_{i}\right) a_{i}^{2}$. Finally, for a given ordered $k$-tuple of marked trees $\widetilde{\mathcal{D}}_{1}, \ldots, \widetilde{\mathcal{D}}_{k}$, the number of possible shuffles is the multinomial coefficient

$$
\left(\begin{array}{c}
2 d \\
2 a_{1}, \ldots, 2 a_{k}
\end{array}\right)=\frac{(2 d) !}{\left(2 a_{1}\right) ! \cdots\left(2 a_{k}\right) !} .
$$

Putting everything together, we obtain 4.6.

Theorem 4.10. The generating function

$$
y(x)=\sum_{d=1}^{\infty} \frac{d^{2} N_{d, 0}((d), \emptyset)}{(2 d) !} x^{d}=\frac{1}{2} x+\frac{1}{6} x^{2}+\frac{7}{80} x^{3}+\frac{23}{420} x^{4}+\cdots
$$

is the unique solution of the initial value problem

$$
x\left(4 y^{\prime}-e^{y}-x e^{y} y^{\prime}\right)=2 y, \quad y(0)=0 .
$$

Proof. Let us define $\tilde{z}(d)=d^{2} z(d) /(2 d)$ ! and $\tilde{z}(0)=0$, so that we have $y(x)=$ $\sum_{d=0}^{\infty} \tilde{z}(d) x^{d}$. The recurrence 4.6 can be rewritten as

$$
\frac{2(2 d+1)}{d+1} \tilde{z}(d+1)=\sum_{k=0}^{\infty} \frac{1}{k !} \sum_{\substack{a_{1}+\cdots+a_{k}=d \\ a_{1}, \ldots, a_{k} \geq 0}} \prod_{i=1}^{k} \tilde{z}\left(a_{i}\right),
$$

which implies

$$
\sum_{d=0}^{\infty}\left(4-\frac{2}{d+1}\right) \tilde{z}(d+1) x^{d+1}=x \sum_{k=0}^{\infty} \frac{1}{k !}(y(x))^{k}=x e^{y} .
$$

Differentiating, we get

$$
4 y^{\prime}-2 \sum_{d=0}^{\infty} \tilde{z}(d+1) x^{d}=4 y^{\prime}-2 \frac{y}{x}=e^{y}+x e^{y} y^{\prime},
$$

and (4.8) follows. 


\subsection{Curves with prescribed tangency at a given point}

Corollary 4.11. The number $N_{d, g}\left((k),\left\langle 1^{d-k}\right\rangle\right)$ of irreducible plane complex algebraic curves of degree $d$ and genus $g$ passing through a generic configuration of $3 d+g-$ $k-1$ points and having tangency of order $k$ to a given line at a given point is equal to $\sum_{\widetilde{\mathcal{D}}} \mu(\widetilde{\mathcal{D}})$, the sum over marked floor diagrams $\widetilde{\mathcal{D}}$ of degree $d$ and genus $g$ in which the last $k$ vertices are sinks connected to the same vertex.

Proof. Indeed, the (ordinary) marked floor diagrams of this kind are in multiplicitypreserving bijection with the $\left((k),\left\langle 1^{d-k}\right\rangle\right)$-marked floor diagrams: simply glue the edges pointing to the last $k$ sinks into a single edge of weight $k$.

Example 4.12. Let us compute (once again) the number $N_{3,0}((2),(1))$. Among the (ordinary) marked floor diagrams of degree $d=3$ and genus $g=0$, there are exactly two in which the last two sinks are not connected to the same vertex; they are shown in Figure 9 Each of the two has multiplicity 1 , and we conclude that $N_{3,0}((2),(1))=N_{3,0}-2=$ $12-2=10$, in agreement with Figure 7

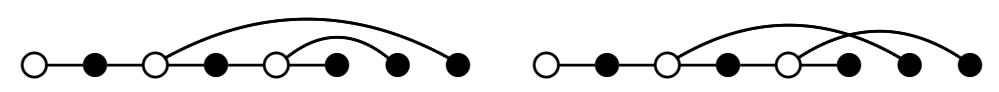

Fig. 9. Marked floor diagrams not contributing to $N_{3,0}((2),(1))$.

\section{Node polynomials}

This section is devoted to the classical problem of determining the Severi degrees $N^{d, \delta}$ (see Section 1.2 when the cogenus $\delta$ is fixed. In other words, how does the number $N^{d, \delta}$ of $\delta$-nodal (possibly reducible) plane curves depend on the degree $d$ ? As already noted in (1.6), if $d$ is large enough (specifically $d \geq \delta+2$ ), then all curves counted by $N^{d, \delta}$ are irreducible, and the Severi degree coincides with the corresponding Gromov-Witten invariant: $N^{d, \delta}=N_{d,(d-1)(d-2) / 2-\delta}$.

Substantial efforts have been expended by various researchers to determine $N^{d, \delta}$, as a function of $d$, for specific small values of $\delta$; see [22, Remark 3.7] for a thorough historical review. For $\delta \leq 3$, the formulas go back to the 19th century (J. Steiner, A. Cayley, G. Salmon, and S. Roberts; see the references in [22]). In particular:

$$
\begin{aligned}
& N^{d, 0}=1, \\
& N^{d, 1}=3(d-1)^{2}, \\
& N^{d, 2}=\frac{3}{2}(d-1)(d-2)\left(3 d^{2}-3 d-11\right) .
\end{aligned}
$$

For $\delta \leq 6$, the problem has been solved by I. Vainsencher [41]; see [14, Proposition 2] for explicit formulas. This has been extended to $\delta \leq 8$ by S. Kleiman and R. Piene [22] by further refining Vainsencher's method; see Remark 5.4 below. Other approaches to 
computing $N^{d, \delta}$ when $\delta$ is small were developed by J. Harris and R. Pandharipande [20], L. Göttsche [19], and Y. Choi [12, 13] (based on the work of Z. Ran [32, 33]).

The following polynomiality property has been first suggested by P. Di Francesco and C. Itzykson [14, Remark (b) after Proposition 2], and then stated as a special case of a more general conjecture by L. Göttsche [19, Conjecture 4.1 and Remark 4.2(2)].

Theorem 5.1. For any fixed $\delta$, there exist a polynomial $N_{\delta}(d) \in \mathbb{Q}[d]$ of degree $2 \delta$ and a threshold value $d_{0}(\delta)$ such that for $d \geq d_{0}(\delta)$, we have $N^{d, \delta}=N_{\delta}(d)$.

Besides establishing polynomiality, our proof provides a method (admittedly tortuous) for computing the polynomials $N_{\delta}(d)$. Cf. Remark 5.5

Remark 5.2. We prove Theorem 5.1 with $d_{0}(\delta)=2 \delta$. By further refining the argument, F. Block [6] has recently improved this to $d_{0}(\delta)=\delta$.

L. Göttsche formulated his conjecture with $d_{0}(\delta)=\lceil\delta / 2\rceil+1$. This was verified by Block for all $\delta \leq 13$ (cf. Remark 5.5). P. Di Francesco and C. Itzykson seem to suggest the threshold value $d_{0}(\delta)=3 / 2+\sqrt{2 \delta+1 / 4}$ (so that $d \geq d_{0}(\delta)$ is equivalent to $\delta \leq(d-1)(d-2) / 2)$. Block's computations show this fails, for the first time, for $\delta=13$.

Remark 5.3. According to Y. Choi ([13, Section 3], unpublished; see citations in [22, Remark 3.7] and [19, Remark 4.2(2)]), Theorem 5.1, with $d_{0}(\delta)=\delta$, can be deduced from [32, Theorem 5].

In an unpublished preprint [26] (cf. also [25]), A.-K. Liu put forward a proof of polynomiality of Severi degrees, in a more general setting of counting curves on an arbitrary surface.

Another proof of this result has been recently announced by Y.-J. Tzeng [40].

Remark 5.4. In the terminology of S. Kleiman and R. Piene [22], $N_{\delta}(d)$ is called a node polynomial. The node polynomials $N_{0}(d), N_{1}(d)$, and $N_{2}(d)$ are given by 5.1 , 5.2), and 5.3 , respectively. The corresponding minimal threshold values $d_{0}(\delta)$ are all equal to 1 .

Kleiman and Piene [22, Section 3] computed the node polynomials $N_{\delta}(d)$ for $\delta \leq 8$, thereby establishing the corresponding instances of Göttsche's conjecture (Theorem 5.1]. Their computations can be summarized as follows:

The generating function for the node polynomials $N_{\delta}(d)$ is given by

$$
\sum_{\delta \geq 0} N_{\delta}(d) t^{\delta}=\exp \left(\sum_{j \geq 1} \frac{A_{j}(d)}{j} t^{j}\right),
$$

where

$$
\begin{aligned}
& A_{1}(d)=3(d-1)^{2}, \\
& A_{2}(d)=-3(d-1)(14 d-25), \\
& A_{3}(d)=3\left(230 d^{2}-788 d+633\right) \\
& A_{4}(d)=9\left(-1340 d^{2}+5315 d-5023\right), \\
& A_{5}(d)=9\left(24192 d^{2}-107294 d+114647\right),
\end{aligned}
$$




$$
\begin{aligned}
& A_{6}(d)=9\left(-445592 d^{2}+2161292 d-2545325\right), \\
& A_{7}(d)=54\left(1386758 d^{2}-7245004 d+9242081\right), \\
& A_{8}(d)=9\left(-156931220 d^{2}+873420627 d-1191950551\right) .
\end{aligned}
$$

For $\delta \leq 8$, the polynomials $N_{\delta}(d)$ computed via (5.4) yield correct values of all nonvanishing Severi degrees $N^{d, \delta}$, and even some zero values, namely $N^{1,1}=N^{1,2}=N^{2,2}=$ $N^{3,4}=0$.

Remark 5.5. Our method for computing the node polynomials was implemented, with substantial algorithmic improvements, by F. Block [6], and used to compute $N_{\delta}(d)$ for all $\delta \leq 13$. Block's calculations confirmed that the polynomials $A_{j}(d)$ defined by 5.4 are indeed quadratic in $d$ (for $\delta \leq 13$ ), in agreement with the strong form of Göttsche's conjecture.

Proof of Theorem 5.1 Our proof is purely combinatorial, and directly based on Theorem 1.6

Let us call an edge $e$ in a labeled floor diagram $\mathcal{D}$ short if $e$ has weight 1 , and connects consecutive vertices. If $\mathcal{D}$ has small cogenus $\delta$, then "almost all" edges in $\mathcal{D}$ are short. Removing those edges and considering the "components" of what remains, we arrive at the following notion, which will play a key role in the proof.

Definition 5.6 (Templates). A template $\Gamma$ is a finite nonempty collection of weighted edges on a finite linearly ordered vertex set $\left\{v_{0}<v_{1}<\cdots<v_{\ell}\right\}$ such that

- for each edge $v_{i} \stackrel{e}{\longrightarrow} v_{j}$ in $\Gamma$, we have $i<j$;

- the weight $w(e)$ of every edge $e$ in $\Gamma$ is a positive integer;

- the weight of an edge of the form $v_{i} \rightarrow v_{i+1}$ must be $\geq 2$ ("no short edges");

- multiple edges are allowed, but loops are not;

- for every $j \in\{1, \ldots, \ell-1\}$, there is at least one edge $v_{i} \stackrel{e}{\longrightarrow} v_{k}$ with $i<j<k$.

With a template $\Gamma$, we associate several quantities. The number $\ell=\ell(\Gamma)$ is called the length of $\Gamma$. The product of squares of edge weights is the multiplicity of $\Gamma$, denoted by $\mu(\Gamma)$; cf. 1.2$)$. The number

$$
\delta(\Gamma)=\sum_{v_{i} \stackrel{e}{\longrightarrow} v_{j}}((j-i) w(e)-1)
$$

is the cogenus of $\Gamma$. (The terminology will be justified by Lemma 5.7) We set

$$
\varepsilon(\Gamma)= \begin{cases}1 & \text { if all edges arriving at } v_{\ell} \text { have weight } 1 ; \\ 0 & \text { otherwise }\end{cases}
$$

For $j \in\{1, \ldots, \ell\}$, let $\varkappa_{j}=\varkappa_{j}(\Gamma)$ denote the total weight of all edges $v_{i} \stackrel{e}{\longrightarrow} v_{k}$ with $i<j \leq k$. By definition of a template, we have $\varkappa_{j}>0$. Let $\varkappa(\Gamma)=\left(\varkappa_{1}, \ldots, \varkappa_{\ell}\right)$. Set

$$
k_{\min }(\Gamma)=\max _{1 \leq j \leq \ell}\left(\varkappa_{j}-j+1\right) .
$$

Figure 10 shows all templates $\Gamma$ with $\delta(\Gamma)=1$ or $\delta(\Gamma)=2$, and the respective values of $\delta(\Gamma), \ell(\Gamma), \mu(\Gamma), \varepsilon(\Gamma), \varkappa(\Gamma)$, and $k_{\min }(\Gamma)$. 


\begin{tabular}{|c|c|c|c|c|c|c|c|}
\hline$\Gamma$ & $\delta(\Gamma)$ & $\ell(\Gamma)$ & $\mu(\Gamma)$ & $\varepsilon(\Gamma)$ & $\varkappa(\Gamma)$ & $k_{\min }(\Gamma)$ & $P(\Gamma, k)$ \\
\hline 0 & 1 & 1 & 4 & 0 & (2) & 2 & $k-1$ \\
\hline$\sigma$ & 1 & 2 & 1 & 1 & $(1,1)$ & 1 & $2 k+1$ \\
\hline O & 2 & 1 & 9 & 0 & (3) & 3 & $k-2$ \\
\hline $\mathrm{O}_{2}^{2}$ & 2 & 1 & 16 & 0 & (4) & 4 & $\left(\begin{array}{c}k-2 \\
2\end{array}\right)$ \\
\hline$\alpha$ & 2 & 2 & 1 & 1 & $(2,2)$ & 2 & $\left(\begin{array}{c}2 k \\
2\end{array}\right)$ \\
\hline 0 & 2 & 2 & 4 & 1 & $(3,1)$ & 3 & $2 k(k-2)$ \\
\hline $0-\overline{2}$ & 2 & 2 & 4 & 0 & $(1,3)$ & 2 & $2 k(k-1)$ \\
\hline 0 & 2 & 3 & 1 & 1 & $(1,1,1)$ & 1 & $3(k+1)$ \\
\hline $0 \quad 0$ & 2 & 3 & 1 & 1 & $(1,2,1)$ & 1 & $k(4 k+5)$ \\
\hline
\end{tabular}

Fig. 10. Templates with $\delta(\Gamma) \leq 2$.

For the remainder of this proof, we allow disconnected labeled floor diagrams $\mathcal{D}$. The degree $d$ and cogenus $\delta$ of such a diagram are determined from the degrees $d_{j}$ and cogenera $\delta_{j}$ of its connected components by the formulas $(1.7)-(1.8)$. As we noted earlier (see the comment following Corollary 1.9, the Severi degree $N^{d, \delta}$ is obtained by counting the markings of all such diagrams (with the given $d$ and $\delta$ ) with the usual multiplicities $\mu(\mathcal{D})$.

Let $\mathcal{D}$ be a (possibly disconnected) labeled floor diagram of degree $d$ and cogenus $\delta$. It will be convenient to add an extra vertex $d+1$ to the vertices $1, \ldots, d$ of $\mathcal{D}$, and connect each vertex $v$ in $\mathcal{D}$ to this new vertex by $1-\operatorname{div}(v)$ new edges of weight 1 . Let $\mathcal{D}^{\prime}$ denote the resulting diagram. To illustrate, applying this procedure to the labeled floor diagram $\mathcal{D}$ shown in 1.3 results in the diagram $\mathcal{D}^{\prime}$ drawn below:

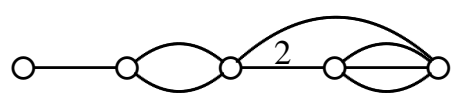

Upon removal of all short edges from $\mathcal{D}^{\prime}$, one obtains a (uniquely defined) collection of nonoverlapping templates. To be pedantic, let $\Gamma_{1}, \ldots, \Gamma_{m}$ be these templates, listed left to right. Denoting the leftmost vertex of $\Gamma_{i}$ by $k_{i}$ (we call $k_{i}$ the offset of $\Gamma_{i}$ ), we then have

$$
k_{i}+\ell\left(\Gamma_{i}\right) \leq k_{i+1} \quad \text { for } 1 \leq i \leq m-1
$$

(so that the $\Gamma_{i}$ do not overlap), and also

$$
k_{m}+\ell\left(\Gamma_{m}\right) \leq d+\varepsilon\left(\Gamma_{m}\right)
$$

(so that $\Gamma_{m}$ properly fits at the right end). 
For $q \leq d+1$, let $a_{q}$ denote the number of short edges connecting $q-1$ to $q$ in $\mathcal{D}^{\prime}$. The divergence condition implies that in $\mathcal{D}^{\prime}$, the total weight of the edges $p \stackrel{e}{\longrightarrow} r$ with $p<q \leq r$ is precisely $q-1$. Then $q-1-a_{q}$ is the total weight of such edges contained in (one of) the templates $\Gamma_{1}, \ldots, \Gamma_{m}$. If say $q=k_{i}+j$ is located in $\Gamma_{i}$ (so that $q$ corresponds to $v_{j}$ in the notation of Definition 5.6, then we have $\varkappa_{j}=q-1-a_{q}=k_{i}+j-1-a_{q} \leq$ $k_{i}+j-1$, implying (cf. (5.6) that

$$
k_{i} \geq k_{\min }\left(\Gamma_{i}\right) \quad \text { for } 1 \leq i \leq m .
$$

Conversely, given a sequence of isomorphism types of templates $\Gamma_{1}, \ldots, \Gamma_{m}$ and an increasing sequence of positive integers $k_{1}<\cdots<k_{m}$ satisfying the inequalities (5.8) 5.10), there is a unique (possibly disconnected) labeled floor diagram $\mathcal{D}$ whose modification $\mathcal{D}^{\prime}$ is obtained by placing each $\Gamma_{i}$ with an offset $k_{i}$, and adding short edges as needed. Specifically, we add $a_{q}$ edges between $q-1$ and $q$, with $a_{q}$ given by

$$
a_{q}= \begin{cases}q-1-\varkappa_{j} & \text { if } q=k_{i}+j \text { for } 1 \leq i \leq m \text { and } 1 \leq j \leq \ell\left(\Gamma_{i}\right) \\ q-1 & \text { otherwise. }\end{cases}
$$

Lemma 5.7. The cogenus $\delta=\delta(\mathcal{D})$ (as defined by $(1.8)$ ) is equal to

$$
\delta=\sum_{i=1}^{m} \delta\left(\Gamma_{i}\right)
$$

where the numbers $\delta\left(\Gamma_{i}\right)$ are defined by (5.5).

Proof. Note that removing a template $\Gamma_{k}$ from the list would result in replacing each of its edges $v_{i} \stackrel{e}{\longrightarrow} v_{j}$ of weight $w(e)$ by the appropriate collection of short edges

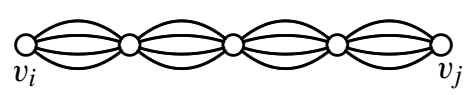

in which each pair of consecutive vertices is connected by $w(e)$ edges. Such a replacement increases the total number of edges by $(j-i) w(e)-1$, thus decreasing the cogenus by the same amount. Since removing all templates would yield a diagram of cogenus 0 , the claim follows.

In order to write down the formula for $N^{d, \delta}$, we will need to keep track of the markings of $\mathcal{D}$ (or of $\mathcal{D}^{\prime}$ ); these break down into markings of individual templates and associated short edges. For a template $\Gamma$ and an offset value $k \in \mathbb{Z}_{>0}$, let $\Gamma_{(k)}$ denote the poset obtained from $\Gamma$ by first adding $k+j-1-\varkappa_{j}$ short edges connecting $j-1$ to $j$ (for $1 \leq j \leq \ell(\Gamma)$; cf. (5.11)), then inserting an extra vertex in the middle of each edge of the resulting graph. Let $P(\Gamma, k)$ denote the number of linear extensions of $\Gamma_{(k)}$, considered modulo automorphisms of $\Gamma_{(k)}$ which fix $\Gamma$ (as in Definition 1.4). With this definition, the number $v(\mathcal{D})$ of markings of a labeled floor diagram $\mathcal{D}$ is given by

$$
\prod_{i=1}^{m} P\left(\Gamma_{i}, k_{i}\right)
$$

where the templates $\Gamma_{i}$ and their respective offsets $k_{i}$ are the same as before. 
Lemma 5.8. For a fixed template $\Gamma$, the values $P(\Gamma, k)$, for $k \geq k_{\min }(\Gamma)$, are given by $a$ polynomial in $k$ whose degree is equal to the number of edges in $\Gamma$.

Proof. First linearly order the vertices of $\Gamma$ together with the midpoints of its edges. For each of these (finitely many) choices, we need to count the number of ways of completing it to a linear extension of $\Gamma_{(k)}$ (here everything is done modulo the appropriate automorphism group). Such a completion amounts to choosing, for each $j \leq \ell(\Gamma)$, a particular shuffle of the $k+j-1-\varkappa_{j}$ (unordered) midpoints of short edges connecting $j-1$ to $j$ with a fixed (i.e., independent of $k$ ) number $b_{j}$ of midpoints of edges of $\Gamma$. Hence the answer is

$$
\prod_{j}\left(\begin{array}{c}
k+j-1-\varkappa_{j}+b_{j} \\
b_{j}
\end{array}\right)
$$

and the claim follows.

Figure 10 shows the polynomials $P(\Gamma, k)$ for the templates $\Gamma$ with $\delta(\Gamma) \leq 2$.

Putting all the ingredients together, we see that the Severi degree $N^{d, \bar{\delta}}$ is given by

$$
N^{d, \delta}=\sum_{m=1}^{\delta} \sum_{\Gamma_{1}, \ldots, \Gamma_{m}}\left(\prod_{i=1}^{m} \mu\left(\Gamma_{i}\right)\right) \sum_{k_{1}, \ldots, k_{m}} \prod_{i=1}^{m} P\left(\Gamma_{i}, k_{i}\right),
$$

where the second sum is over all $m$-tuples of templates $\left(\Gamma_{1}, \ldots, \Gamma_{m}\right)$ satisfying $(5.12)$, and the third sum is over $m$-tuples of integer offsets $\left(k_{1}, \ldots, k_{m}\right)$ satisfying $[5.8-5.10$ ) (This calculation is illustrated for $\delta=2$ in Example 5.9 below.) Now let us write the aforementioned third sum as

$$
\sum_{\substack{k_{m} \geq k_{\min }\left(\Gamma_{m}\right) \\ k_{m} \leq d-\ell\left(\Gamma_{m}\right)+\varepsilon\left(\Gamma_{m}\right)}} P\left(\Gamma_{m}, k_{m}\right) \cdots \sum_{\substack{k_{2} \geq k_{\min }\left(\Gamma_{2}\right) \\ k_{2} \leq k_{3}-\ell\left(\Gamma_{2}\right)}} P\left(\Gamma_{2}, k_{2}\right) \sum_{\substack{k_{1} \geq k_{\min }\left(\Gamma_{1}\right) \\ k_{1} \leq k_{2}-\ell\left(\Gamma_{1}\right)}} P\left(\Gamma_{1}, k_{1}\right) .
$$

If $P(k)$ is given by a polynomial in $k$ for $k \geq c$, and $a$ and $b$ are positive integer constants, then $\sum_{k \geq a, k \leq n-b} P(k)$ is given by a polynomial in $n$ (of one degree higher) for $n \geq$ $\max (a+b, c)$. Iterating this argument, we conclude that $N^{d, \delta}$ is given by a polynomial in $d$ if

$$
d \geq k_{\min }\left(\Gamma_{1}\right)+\ell\left(\Gamma_{1}\right)+\cdots+\ell\left(\Gamma_{m}\right)-\varepsilon\left(\Gamma_{m}\right)
$$

for any allowable choice of $\Gamma_{1}, \ldots, \Gamma_{m}$. It is not hard to see that the degree of the resulting polynomial is $2 \delta$; indeed, the maximal value of $m$ is $\delta$, and the maximal total number of edges in the templates involved is $\delta$ as well. Also, the right-hand side of (5.14) can be seen to be at most $2 \delta$, providing a threshold value.

Example 5.9. For $\delta=2$, substituting the data from Figure 10 into formula [5.13, we obtain

$$
\begin{aligned}
N^{d, 2}= & \sum_{k=2}^{d-1}\left(9(k-2)+16\left(\begin{array}{c}
k-2 \\
2
\end{array}\right)+\left(\begin{array}{c}
2 k \\
2
\end{array}\right)+8 k(k-2)\right) \\
& +\sum_{k=1}^{d-2}(8 k(k-1)+3(k+1)+k(4 k+5))
\end{aligned}
$$




$$
\begin{aligned}
& +\sum_{k_{2} \leq d-1} \sum_{k_{1} \leq k_{2}-1}\left(16\left(k_{1}-1\right)\left(k_{2}-1\right)+4\left(k_{1}-1\right)\left(2 k_{2}+1\right)\right) \\
& +\sum_{k_{2} \leq d-1} \sum_{k_{1} \leq k_{2}-2}\left(\left(2 k_{1}+1\right) \cdot 4\left(k_{2}-1\right)+\left(2 k_{1}+1\right)\left(2 k_{2}+1\right)\right),
\end{aligned}
$$

which after tedious calculations (or with the help of your favourite software) yields 5.3 .

\section{Enumeration of labeled floor diagrams of genus 0}

Theorem 6.1. The number of labeled floor diagrams of degree $d$ and genus 0 is $d^{d-2}$.

Recall that $d^{d-2}$ is also the number of labeled trees on $d$ vertices, or equivalently the number of trees on the vertex set $\{1, \ldots, d\}$. This classical result is commonly known as Cayley's formula (see for example [24, Theorem 2.1] and [38, pages 25 and 66]).

Proof of Theorem 6.1. Let $\ell(d)$ denote the number of labeled floor diagrams of degree $d$ and genus 0 . Let $t(d)=d^{d-2}$ denote the number of labeled trees on $d$ vertices. Our goal is to show that $\ell(d)=t(d)$.

It is well known, and easy to deduce, that

$$
t(d)=\sum_{k} \sum_{S_{1} \cup \ldots \cup S_{k}=\{1, \ldots, d-1\}} \prod_{i=1}^{k} t\left(\left|S_{i}\right|\right)\left|S_{i}\right|,
$$

where the second sum is over all unordered set partitions of the set $\{1, \ldots, d-1\}$ into $k$ nonempty blocks $S_{1}, \ldots, S_{k}$. (Thus the number of summands is $S(d-1, k)$, the Stirling number of the second kind [37, Section 1.4].) Formula 6.1] is an enumerative encoding of the decomposition of a labeled tree into a root vertex $d$ and a collection of disjoint subtrees attached to $d$, whose vertex sets form a set partition of $\{1, \ldots, d-1\}$.

Together with the initial condition $t(1)=1$, the recurrence (6.1) uniquely determines the sequence $t(d)$. Hence the theorem will follow if we show that the numbers $\ell(d)$ satisfy the same recurrence. This can be established in the manner analogous to the proof of 6.1 given above. Every labeled floor diagram $\mathcal{D}$ of genus 0 and degree $d$ is a particular kind of a weighted labeled tree on the vertex set $\{1, \ldots, d\}$. As such, it can be disassembled into the root vertex $d$ and an unordered collection of genus-0 floor diagrams $\mathcal{D}_{1}, \ldots, \mathcal{D}_{k}$ supported on disjoint nonempty vertex sets $S_{1}, \ldots, S_{k}$ and joined to $d$ by weighted edges. There is exactly one such weighted edge connecting $d$ to each set $S_{i}$. It remains to check that for a given labeled floor diagram $\mathcal{D}_{i}$ on a vertex set $S_{i} \subset\{1, \ldots, d-1\}$, there are exactly $\left|S_{i}\right|$ ways to connect a vertex $v \in S_{i}$ to $d$ by a weighted edge $v \stackrel{e}{\longrightarrow} d$ so that the resulting weighted tree on $\left|S_{i}\right|+1$ vertices is a labeled floor diagram. (Gluing those diagrams together will produce a labeled floor diagram on $\{1, \ldots, d\}$.) To prove this, note that for a fixed $v$, the only restriction on the weight $w(e)$ comes from the divergence condition 1.1 . The latter implies that $w(e)$ can be chosen to be any positive 
integer not exceeding $1-\operatorname{div}_{i}(v)$, where $\operatorname{div}_{i}(v)$ denotes the divergence at $v$ within the subdiagram $\mathcal{D}_{i}$. It follows that the number of choices in question is

$$
\sum_{v \in S_{i}}\left(1-\operatorname{div}_{i}(v)\right)=\left|S_{i}\right|,
$$

as desired.

The proof of Theorem 6.1 given above can be used to construct explicit bijections between labeled floor diagrams of genus 0 and degree $d$, on one hand, and labeled trees on $d$ vertices, on the other. One family of bijections of this kind, illustrated in Appendix A, is built recursively as follows.

Definition 6.2 (A bijection between labeled floor diagrams and labeled trees). Suppose we have already defined such bijections for labeled floor diagrams of genus 0 and any degree $<d$. Now, for a diagram $\mathcal{D}$ of degree $d$, do the following. Decompose $\mathcal{D}$ into the root vertex $d$ and subdiagrams $\mathcal{D}_{1}, \ldots, \mathcal{D}_{k}$ on vertex sets $S_{1}, \ldots, S_{k}$, as described in the proof of Theorem 6.1 above. Let $T_{1}, \ldots, T_{k}$ denote the trees on vertex sets $S_{1}, \ldots, S_{k}$ that correspond to $\mathcal{D}_{1}, \ldots, \mathcal{D}_{k}$, respectively, under the appropriate bijections. The tree $T$ associated to $\mathcal{D}$ under the bijection in question is constructed by connecting each tree $T_{i}$ to the root vertex $d$ by a single edge $e^{\prime}$ that is going to be determined by the unique weighted edge $v \stackrel{e}{\longrightarrow} d$ connecting $\mathcal{D}_{i}$ to $d$ in $\mathcal{D}$. It remains to describe the rule that determines $e^{\prime}$ from $e$. We have already checked (see $(6.2)$ ) that the total number of choices for $e$ is equal to $\left|S_{i}\right|$. Let us record those choices in an ordered list as the vertex $v$ moves left to right within $\mathcal{D}_{i}$; for a given $v$, we record the choices starting with the largest possible weight value $w(e)=1-\operatorname{div}_{i}(v)$ and decreasing it until we reach the smallest possible weight value $w(e)=1$. We similarly list the $\left|S_{i}\right|$ choices available for the edge $v^{\prime} \stackrel{e^{\prime}}{\longrightarrow} d$ connecting $S_{i}$ to $d$; the ordering is determined by the (left-to-right) ordering of the vertices $v^{\prime} \in S_{i}$. We finally match the choices on both lists in the order they are listed.

Example 6.3. To illustrate, consider the labeled floor diagram $\mathcal{D}_{i}$ shown below alongside with the tree $T_{i}$ associated to it:

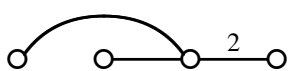

$\mathcal{D}_{i}$

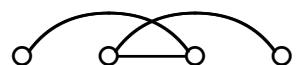

$T_{i}$

There are four ways to augment $\mathcal{D}_{i}$ by a single weighted edge $v \stackrel{e}{\longrightarrow} d$ (here $d$ is a vertex located to the right of $\mathcal{D}_{i}$ ) so that the resulting tree on five vertices is a valid labeled floor diagram. These four possibilities are shown in the first column of Figure 11. Similarly, there are four possibilities, shown in the second column, to connect $T_{i}$ to such a vertex $d$ by a single edge. The bijection matches each labeled floor diagram to the labeled tree shown in the same row of the table.

Lemma 6.4. Let $\mathcal{D}$ be a labeled floor diagram of genus 0 and degree $d$, and let $T$ be the tree on the vertex set $\{1, \ldots, d\}$ associated to it by the bijection described in Definition 6.2 Then, for any $i \in\{1, \ldots, d-1\}$, the following are equivalent: 


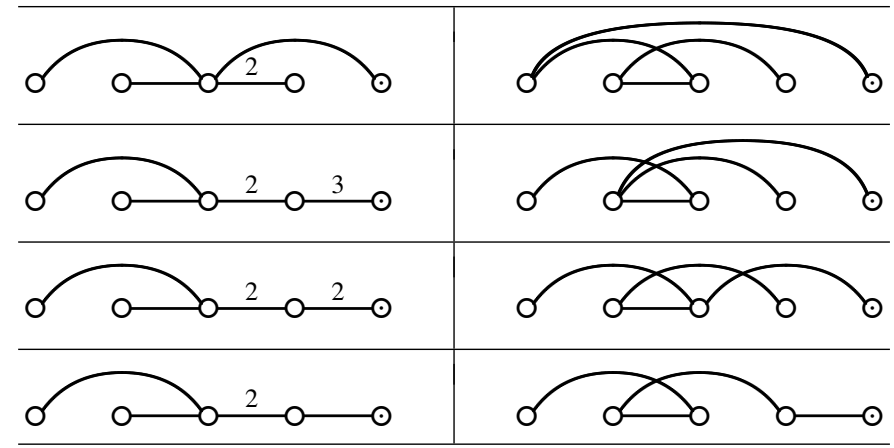

Fig. 11. Building a bijection between labeled floor diagrams and trees.

- $\mathcal{D}$ contains an edge $i \rightarrow i+1$, and this edge has weight 1 ;

- $T$ contains the edge $(i, i+1)$.

Proof. This lemma is a consequence of the following observation: in the ordered lists of choices involved in a recursive step of the bijection described in Definition 6.2, the last elements are:

- the edge of weight 1 connecting the rightmost vertex in $\mathcal{D}_{i}$ to $d$;

- the edge connecting the rightmost vertex in $T_{i}$ to $d$.

Cf. for example the last row in Figure 11.

Lemma 6.4 directly implies the following enumerative corollary.

Corollary 6.5. For any subset $A \subset\{1, \ldots, d-1\}$, the following are equal:

- the number of labeled floor diagrams of genus 0 and degree $d$ in which all the edges of the form $a \rightarrow a+1$ for $a \in A$ are present, each with weight 1 ;

- the number of trees on the vertex set $\{1, \ldots, d\}$ containing all the edges of the form $(a, a+1)$ for $a \in A$.

As an application of Corollary 6.5, we obtain:

Corollary 6.6. For a given $a \in\{1, \ldots, d-1\}$, the number of labeled floor diagrams of genus 0 and degree $d$ containing an edge $a \rightarrow a+1$, with weight 1 , is equal to $2 d^{d-3}$. In particular, this number does not depend on $a$.

More generally, for $1 \leq a<a+b \leq d$, the number of labeled floor diagrams of genus 0 and degree $d$ which contain the edges $a \rightarrow a+1 \rightarrow \cdots \rightarrow a+b$, all with weight 1 , is equal to $(b+1) d^{d-b-2}$.

Proof. By Corollary 6.5, the quantity in question is equal to the number of trees on the vertex set $\{1, \ldots, d\}$ containing all the edges $(a, a+1),(a+1, a+2), \ldots,(a+b-1, a+b)$. Equivalently, this is the number of spanning trees in the graph obtained from the $d$-vertex complete graph $K_{d}$ by contracting all edges connecting some $b+1$ vertices to each other. 
Computing the number of such spanning trees is a straightforward application of the Matrix-Tree Theorem (see, e.g., [24, Theorem 36.1] or [38, Theorem 5.6.8]), which is left to the reader.

Another curious enumerative result concerning labeled floor diagrams is the following byproduct of our proof of Theorem 4.9

Proposition 6.7. The number of labeled floor diagrams of genus 0 and degree d containing an edge e of weight $w(e)=d-1$ is equal to $(d-2)$ !.

Proof. These labeled floor diagrams are in bijection with increasing rooted trees on $d-1$ vertices (see the proof of Theorem 4.9). The number of such trees is well known to be equal to $(d-2)$ !; see, e.g., [37, Proposition 1.3.16].

It is easy to see from Definition 1.1 that $d-1$ is the largest possible edge weight in a floor diagram of degree $d$.

\section{Conjectures and open problems}

\subsection{Higher genera}

One would obviously like to extend Theorem 6.1 beyond the case $g=0$.

Problem 7.1. Enumerate labeled floor diagrams of degree $d$ and genus $g>0$.

At this moment, we do not have a conjectural formula for the number $l_{d, g}$ of such diagrams. Using the data in Appendix A, one concludes that

$$
l_{3,1}=1, l_{4,1}=13, l_{4,2}=5, \quad l_{4,3}=1 .
$$

The rest of this section is devoted exclusively to the case $g=0$.

\subsection{Tree statistics}

Let $\varphi_{d}$ be a bijection that maps a labeled tree $T$ on $d$ vertices to a labeled floor diagram $\mathcal{D}=\varphi_{d}(T)$ of genus 0 and degree $d$; an example of such a bijection was given in Definition 6.2. We can then lift the functions $\mathcal{D} \mapsto \mu(\mathcal{D})$ and $\mathcal{D} \mapsto v(\mathcal{D})$ to the corresponding tree statistics

$$
T \mapsto \hat{\mu}(T)=\mu\left(\varphi_{d}(T)\right), \quad T \mapsto \hat{v}(T)=v\left(\varphi_{d}(T)\right)
$$

which are obviously equidistributed with $(\mu, v)$. Then Theorem 1.6 yields a formula for the Gromov-Witten numbers $N_{d, 0}$ in terms of $\hat{\mu}$ and $\hat{v}$ :

$$
N_{d, 0}=\sum_{T} \hat{\mu}(T) \hat{v}(T)
$$

the sum over all labeled trees $T$ on $d$ vertices.

The tree statistics $\hat{\mu}$ and $\hat{v}$ derived from the bijection of Definition 6.2 turn out to be quite complicated, so the resulting formula (7.1) is not as elegant as one might desire. This naturally leads to the following problem. 
Problem 7.2. Find tree statistics $\hat{\mu}$ and $\hat{v}$, as conceptually simple as possible, whose joint distribution on the set of labeled trees on $d$ vertices coincides with the joint distribution of $\mu$ and $\nu$ on the set of labeled floor diagrams of degree $d$ and genus 0 .

\subsection{Recurrences of Kontsevich and Caporaso-Harris}

As mentioned in [7, Exercice 6.2] (cf. also [2]), it is possible to use Theorem 3.18 to provide a combinatorial derivation of the Caporaso-Harris recurrence, somewhat similar in spirit to the proof given by A. Gathmann and H. Markwig [16].

The celebrated formula of Kontsevich [23, (5.17)] determines the genus-0 GromovWitten invariants $N_{d, 0}$ by means of the recursive relation

$$
N_{d, 0}=\sum_{k+l=d} N_{k, 0} N_{l, 0} k^{2} l\left(l\left(\begin{array}{c}
3 d-4 \\
3 k-2
\end{array}\right)-k\left(\begin{array}{l}
3 d-4 \\
3 k-1
\end{array}\right)\right),
$$

for $d \geq 2$. Even though this recurrence looks much simpler than Caporaso-Harris's, deriving it directly from Theorem 1.6 requires nontrivial effort. The blueprint for such a derivation is provided by Kontsevich's original proof; in order to translate this proof into a combinatorial language, one likely needs to extend the notion of a (labeled) floor diagram to allow for a real parameter, corresponding to the tropical cross-ratio of the appropriate point configuration. While we foresee no insurmountable obstacles to implementing this plan, the technical difficulties involved are substantial enough to require a separate paper.

Problem 7.3. Give a direct proof of Kontsevich's recursion 7.2 based on the combinatorial definition of the numbers $N_{d, 0}$ given by formula (1.6), with $g=0$.

This problem was also posed independently in [7, Exercice 7.2].

\subsection{Alternating trees}

This class of labeled trees was introduced by A. Postnikov et al. [17, 30]; see [39], Section 4] for a survey of related topics. An alternating tree $T$ is a tree on the vertex set $\{1, \ldots, d\}$ such that the vertices adjacent to any given vertex $v$ either all have smaller labels than $v$, or all have larger labels. That is, $T$ must not contain a 3-vertex subtree $a-b-c$ with $a<b<c$.

Let $a_{d}$ denote the number of alternating trees on $d$ vertices. For example, direct inspection of the first table in Appendix A shows that $a_{1}=a_{2}=1, a_{3}=2, a_{4}=7$. Postnikov has shown [30, Theorem 1], [38, Exercise 5.41(b)] that

$$
a_{d}=\frac{1}{d \cdot 2^{d-1}} \sum_{k=1}^{d}\left(\begin{array}{l}
d \\
k
\end{array}\right) k^{d-1}
$$

Problem 7.4. Prove or disprove: The number of trees $T$ on the vertex set $\{1, \ldots, d\}$ for which there is a labeled floor diagram whose underlying tree is $T$ is equal to $a_{d}$. 
In other words, if we ignore edge weights, then the trees obtained from labeled floor diagrams of genus 0 are conjecturally equinumerous to the alternating trees.

A related (but different) enumerative problem is the following.

Problem 7.5. Enumerate multiplicity-free labeled floor diagrams of degree $d$ and genus 0 , i.e., those diagrams in which $w(e)=1$ for every edge $e$.

Our calculations show that for $d=1,2,3,4,5,6$ the number of such diagrams is $1,1,2,7,36,245$, respectively. These values match the first terms of the sequence [36. A029768] (see also [5, Exercise 5.2.20]) that enumerates increasing rooted trees with cyclically ordered branches.

\subsection{Generalizations to other Lie types}

Problem 7.6. Assume that $g=0$. Is there a natural generalization of Theorem 1.6 (including the numbers $N_{d, 0}$ and the notion of a labeled floor diagram) associated with an arbitrary finite indecomposable crystallographic root system $\Phi$ ?

The possibility of such a generalization is prompted by existing interpretations of labeled trees on $d$ vertices (hence, by extension, labeled floor diagrams of genus 0 and degree $d$ ) as "type $A$ objects", i.e., combinatorial gadgets associated with a root system of type $A$. Such an association can actually be made in at least two substantially different ways, involving root systems of types $A_{d-2}$ and $A_{d-1}$, respectively. Both constructions are fairly well known, so we present them cursorily, referring the reader to relevant sources for further details.

Let $\Phi_{>0}$ denote the set of positive roots in the root system $\Phi$. The first construction is based on the notion of the Shi arrangement, the arrangement of hyperplanes in the (co-)root space of $\Phi$ defined by the equations

$$
\langle x, \alpha\rangle \in\{0,1\}, \quad \alpha \in \Phi_{>0} .
$$

It was conjectured by R. W. Carter and proved by J.-Y. Shi [35, Theorem 8.1] that the number of regions of this arrangement (i.e., the number of connected components in the complement to the union of these hyperplanes) is equal to $(h+1)^{n}$, where $n$ is the rank of $\Phi$ and $h$ is the Coxeter number. If $\Phi$ is of type $A_{d-2}$, then $n=d-2$ and $h=d-1$, so $(h+1)^{n}=d^{d-2}$, the number of labeled trees on $d$ vertices. Explicit bijections are known (see [39, Section 5] and [4]) that identify such trees, and therefore labeled floor diagrams of genus 0 and degree $d$, with the regions of the Shi arrangement of type $A_{d-2}$. It is not unreasonable to anticipate a generalization of this correspondence to suitably defined labeled floor diagrams of other types.

It is worth mentioning that the alternating trees discussed earlier in this section have a natural analogue for any root system $\Phi$, since they are equinumerous to (and can be identified with) the regions of the Linial arrangement

$$
\langle x, \alpha\rangle=1, \quad \alpha \in \Phi_{>0} .
$$


(This was conjectured by R. Stanley and proved by A. Postnikov [30, Section 4.2], [31, Theorem 8.2], [38, Exercise 5.41(h)] and later by C. Athanasiadis [3, Theorem 4.1].)

The second construction involves the noncrossing partition lattice $\mathrm{NC}(\Phi)$ associated with the root system $\Phi$. By a theorem of F. Chapoton [11, Proposition 9], the number of maximal chains in $\mathrm{NC}(\Phi)$ is equal to $h^{n} n ! /|W|$, where $W$ denotes the associated Weyl group. For $\Phi$ of type $A_{d-1}$, one recovers Cayley's formula.

\section{Welschinger invariants and odd floor diagrams}

The Gromov-Witten number $N_{d, 0}$ has a "real" counterpart, the Welschinger invariant $W_{d}$ [43, 44] that counts real rational curves of degree $d$ through generic $3 d-1$ points on the real projective plane, each with a certain sign. To be specific, to a nodal algebraic curve in $\mathbb{R P}^{2}$, let us associate a sign that equals $(-1)$ to the power of the number of its solitary nodes (i.e., points locally given by $x^{2}+y^{2}=0$ ). These signs were introduced by Welschinger [44] who proved that the signed count $W_{d}$ does not depend on the choice of a configuration.

The following result is a restatement of (a part of) [8, Theorem 2].

Theorem 8.1. The Welschinger invariant $W_{d}$ is equal to

$$
W_{d}=\sum_{\mu(\mathcal{D}) \equiv 1 \bmod 2} v(\mathcal{D}),
$$

the sum over all labeled floor diagrams $\mathcal{D}$ of degree $d$ and genus 0 whose edge weights are all odd.

Example 8.2. For $d=3$ and $d=4$ (cf. Appendix A), formula (8.1) gives

$$
\begin{aligned}
& W_{3}=5+3=8, \\
& W_{4}=40+35+45+3+24+46+32+15=240 .
\end{aligned}
$$

A labeled floor diagram $\mathcal{D}$ is called odd if the weight $w(e)$ of every edge $e$ in $\mathcal{D}$ is an odd number. Thus, the summation in 8.1 is over all odd labeled floor diagrams $\mathcal{D}$ of degree $d$ and genus 0 .

Problem 8.3. Enumerate the odd labeled floor diagrams of degree $d$ and genus 0 .

Let $b_{d}$ denote the number of such diagrams. Our calculations show that

$$
b_{1}=1, \quad b_{2}=1, \quad b_{3}=2, \quad b_{4}=8, \quad b_{5}=46, \quad b_{6}=352 .
$$

Curiously, these numbers match the first terms of the sequence [36, A099765] given by

$$
b_{d}=\frac{1}{d} \sum_{k=0}^{\lfloor d / 2\rfloor}(-1)^{k}\left(\begin{array}{l}
d \\
k
\end{array}\right)(d-2 k)^{d-1} .
$$

Theorem 8.1 was recently used in [2] to obtain a Caporaso-Harris-type recurrence for Welschinger invariants. 
Appendix A: Labeled floor diagrams with $d \leq 4$

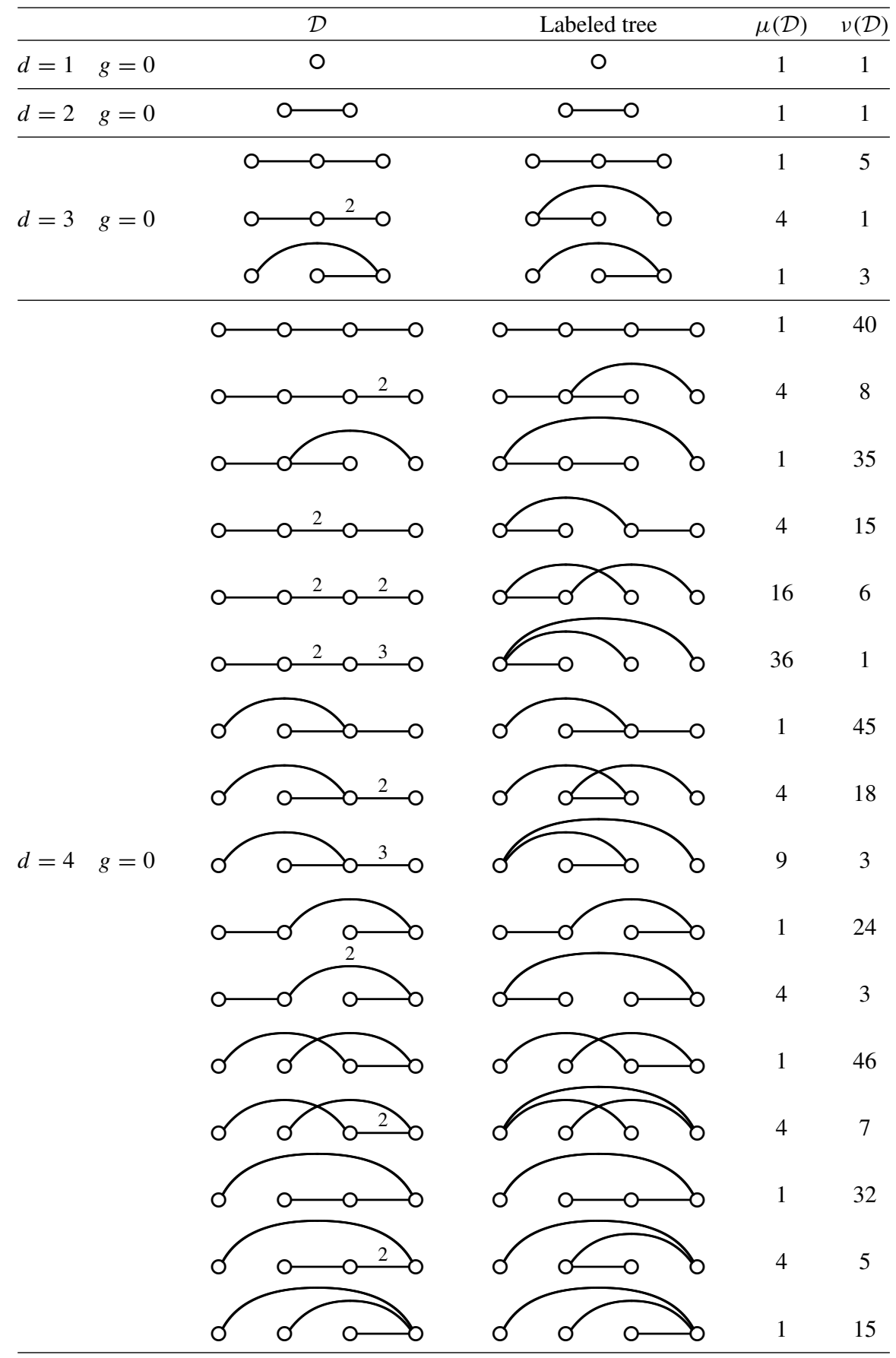



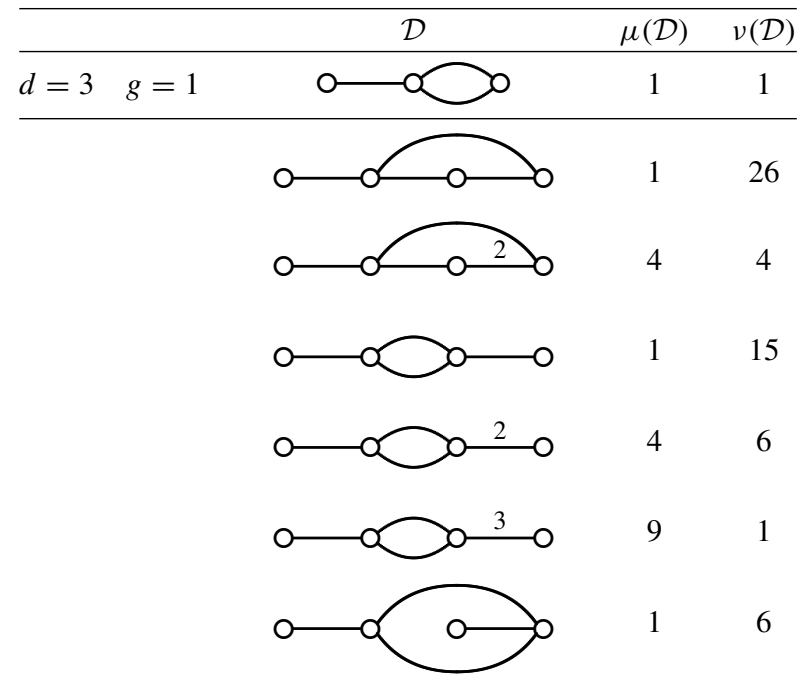

$d=4 \quad g=1 \quad 0 \quad 0001019$
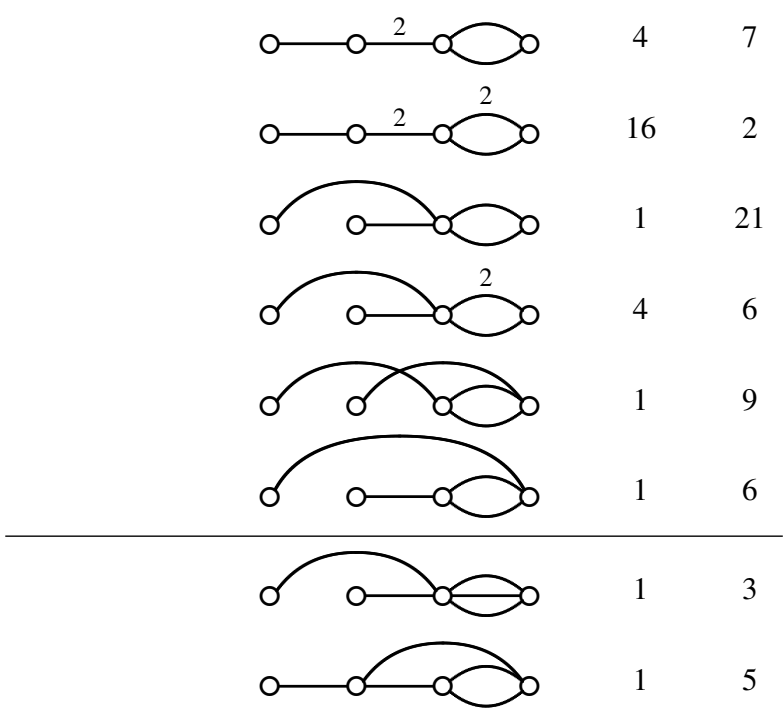

$d=4 \quad g=2 \quad$ OCO $17 \begin{array}{lll}7 & 1\end{array}$

d=4


Appendix B: Tropical rational cubics and their marked floor diagrams

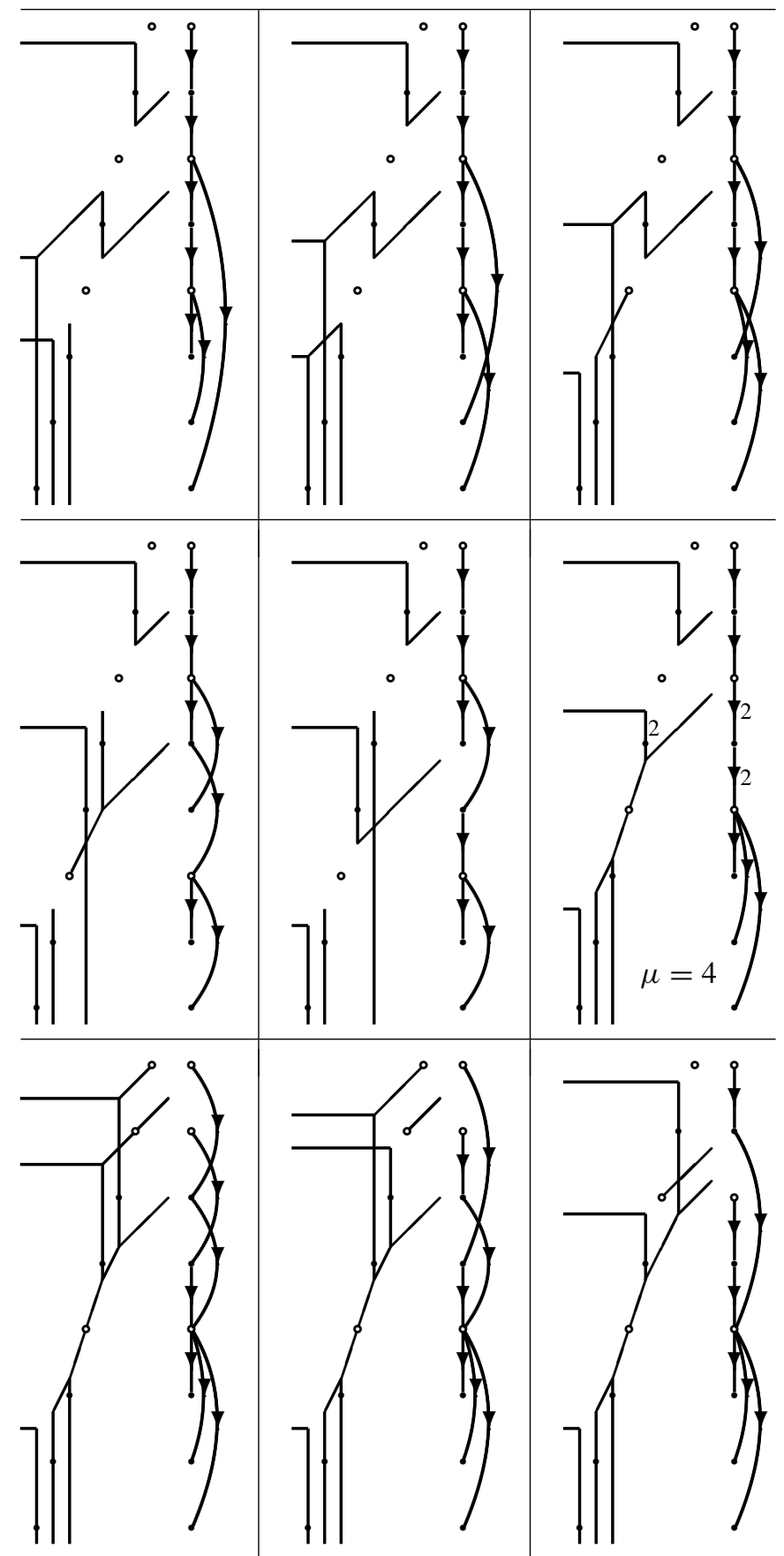


Acknowledgments. We thank Florian Block, Philippe Di Francesco, Bill Fulton, Rahul Pandharipande, Ragni Piene, Michael Shapiro, Alek Vainshtein, and Ravi Vakil for valuable comments.

This research was partially supported by NSF grant DMS-0555880 (S.F.), by SNSF grants 125070 and 126817 and by the TROPGEO project of the European Research Council (G.M.).

\section{References}

[1] Aharony, O., Hanany, A., Kol, B.: Webs of $(p, q)$ 5-branes, five dimensional field theories and grid diagrams. J. High Energy Phys. 9801, 002 (1998)

[2] Arroyo, A., Brugallé, E., López de Medrano, L.: Recursive formulas for Welschinger invariants of the projective plane. Int. Math. Res. Notices, to appear; http://imrn.oxfordjournals.org/ cgi/reprint/rnq096v1

[3] Athanasiadis, C. A.: Characteristic polynomials of subspace arrangements and finite fields. Adv. Math. 122, 193-233 (1996) Zbl 0872.52006 MR 1409420

[4] Athanasiadis, C. A., Linusson, S.: A simple bijection for the regions of the Shi arrangement of hyperplanes. Discrete Math. 204, 27-39 (1999) Zbl 0959.52019 MR 1691861

[5] Bergeron, F., Labelle, G., Leroux, P.: Combinatorial Species and Tree-Like Structures. Cambridge Univ. Press (1998) Zbl 0888.05001 MR 1629341

[6] Block, F.: Computing node polynomials for plane curves. arXiv:1006.0218

[7] Brugallé, E.: Géométries enumératives complexe, réelle et tropicale. In: Géométrie tropicale, Éd. Éc. Polytech., Palaiseau, 27-84 (2008) Zbl 1165.14003 MR 2509158

[8] Brugallé, E., Mikhalkin, G.: Enumeration of curves via floor diagrams. C. R. Math. Acad. Sci. Paris 345, 329-334 (2007) Zbl 1124.14047 MR 2359091

[9] Brugallé, E., Mikhalkin, G.: Floor decomposition of tropical curves: the planar case. In: Proc. 15th Gökova Geometry-Topology Conf. (Gökova, 2008), Int. Press, Cambridge, MA, 64-90 (2009) Zbl pre05575865 MR 2500574

[10] Caporaso, L., Harris, J.: Counting plane curves of any genus. Invent. Math. 131, 345-392 (1998) Zbl 0934.14040 MR 1608583

[11] Chapoton, F.: Enumerative properties of generalized associahedra. Sém. Lothar. Combin. 51, art. B51b, 16 pp. (2004/05) Zbl 1160.05342 MR 2080386

[12] Choi, Y.: Severi degrees in cogenus 4. arXiv:alg-geom/9601013v2

[13] Choi, Y.: On the degree of Severi varieties. Preprint (1997)

[14] Di Francesco, P., Itzykson, C.: Quantum intersection rings. In: The Moduli Space of Curves, Birkhäuser, 81-148 (1995); arXiv:hep-th/9412175v1 Zbl 0868.14029 MR 1363054

[15] Gathmann, A.: Topological recursion relations and Gromov-Witten invariants in higher genus. arXiv:math/0305361v1.

[16] Gathmann, A., Markwig, H.: The Caporaso-Harris formula and plane relative GromovWitten invariants in tropical geometry. Math. Ann. 338, 845-868 (2007) Zbl 1128.14040 MR 2317753

[17] Gelfand, I. M., Graev, M. I., Postnikov, A.: Combinatorics of hypergeometric functions associated with positive roots. In: Arnold-Gelfand Mathematical Seminars: Geometry and Singularity Theory, Birkhäuser, Boston, 205-221 (1997) Zbl 0876.33011 MR 1429893

[18] Gelfand, I. M., Kapranov, M. M., Zelevinsky, A. V.: Discriminants, Resultants, and Multidimensional Determinants. Birkhäuser (1994) Zbl 0827.14036 MR 1264417

[19] Göttsche, L.: A conjectural generating function for numbers of curves on surfaces. Comm. Math. Phys. 196, 523-533 (1998) Zbl 0934.14038 MR 1645204

[20] Harris, J., Pandharipande, R.: Severi degrees in cogenus 3. arXiv:alg-geom/9504003v1 
[21] Itenberg, I., Mikhalkin, G., Shustin, E.: Tropical Algebraic Geometry. Birkhäuser (2007) Zbl 1162.14300 MR 2292729

[22] Kleiman, S. L., Piene, R.: Node polynomials for families: methods and applications. Math. Nachr. 271, 69-90 (2004) Zbl 1066.14063 MR 2068884

[23] Kontsevich, M., Manin, Yu.: Gromov-Witten classes, quantum cohomology, and enumerative geometry. Comm. Math. Phys. 164, 525-562 (1994) Zbl 0853.14020 MR 1291244

[24] van Lint, J. H., Wilson, R. M.: A Course in Combinatorics. 2nd ed., Cambridge Univ. Press (2001) Zbl 0980.05001 MR 1871828

[25] Liu, A.-K.: Family blowup formula, admissible graphs and the enumeration of singular curves. I. J. Differential Geom. 56, 381-579 (2000) Zbl 1036.14014 MR 1868322

[26] Liu, A.-K.: The algebraic proof of the universality theorem. arXiv:math/0402045v1

[27] Mikhalkin, G.: Enumerative tropical algebraic geometry in $\mathbb{R}^{2}$. J. Amer. Math. Soc. 18 (2005), 313-377. Zbl 1092.14068 MR 2137980

[28] Mikhalkin, G.: Tropical geometry and its applications. In: International Congress of Mathematicians (Madrid, 2006), Vol. II, Eur. Math. Soc., Zürich, 827-852 (2006) Zbl 1103.14034 MR 2275625

[29] Mikhalkin, G.: Phase-tropical curves in $\mathbb{R}^{n}$. In preparation

[30] Postnikov, A.: Intransitive trees. J. Combin. Theory Ser. A 79, 360-366 (1997) Zbl 0876.05042 MR 1462563

[31] Postnikov, A., Stanley, R. P.: Deformations of Coxeter hyperplane arrangements. J. Combin. Theory Ser. A 91, 544-597 (2000) Zbl 0962.05004 MR 1780038

[32] Ran, Z.: Enumerative geometry of singular plane curves. Invent. Math. 97, 447-465 (1989) Zbl 0702.14040 MR 1005002

[33] Ran, Z.: On the quantum cohomology of the plane, old and new, and a $K 3$ analogue. Collect. Math. 49, 519-526 (1998) Zbl 0974.14039 MR 1677096

[34] Sagan, B.: The Symmetric Group. Springer (2001) Zbl 0964.05070 MR 1824028

[35] Shi, J.-Y.: Sign types corresponding to an affine Weyl group. J. London Math. Soc. (2) 35, 56-74 (1987) Zbl 0681.20033 MR 0871765

[36] Sloane, N. J. A.: The on-line encyclopedia of integer sequences. http://www.research.att.com rnjas/sequences/ Zbl 1044.11108 MR 1992789

[37] Stanley, R. P.: Enumerative Combinatorics, Vol. 1. Cambridge Univ. Press (1997) Zbl 0889.05001 MR 1442260

[38] Stanley, R. P.: Enumerative Combinatorics, Vol. 2. Cambridge Univ. Press (1999) Zbl 0928.05001 MR 1676282

[39] Stanley, R. P.: Hyperplane arrangements, interval orders, and trees. Proc. Nat. Acad. Sci. U.S.A. 93, 2620-2625 (1996) Zbl 0848.05005 MR 1379568

[40] Tzeng, Y.-J.: A proof of the Göttsche-Yau-Zaslow formula. Preliminary draft (2009)

[41] Vainsencher, I.: Enumeration of $n$-fold tangent hyperplanes to a surface. J. Algebraic Geom. 4, 503-526 (1995) Zbl 0928.14035 MR 1325790

[42] Vakil, R.: Counting curves on rational surfaces. Manuscripta Math. 102, 53-84 (2000) Zbl 0967.14036 MR 1771228

[43] Welschinger, J.-Y.: Invariants of real rational symplectic 4-manifolds and lower bounds in real enumerative geometry. C. R. Math. Acad. Sci. Paris 336, 341-344 (2003) Zbl 1042.57018 MR 1976315

[44] Welschinger, J.-Y.: Invariants of real symplectic 4-manifolds and lower bounds in real enumerative geometry. Invent. Math. 162, 195-234 (2005) Zbl 1082.14052 MR 2198329 